\title{
Reliability Analysis of Large-Scale Adaptive Weighted Networks
}

\author{
Bo Song, Xu Wang, Wei Ni, Senior Member, IEEE, Yurong Song, Ren Ping Liu, Senior Member, IEEE, \\ Guo-Ping Jiang, Senior Member, IEEE, and Y. Jay Guo, Fellow, IEEE
}

\begin{abstract}
Disconnecting impaired or suspicious nodes and rewiring to those reliable, adaptive networks have the potential to inhibit cascading failures, such as DDoS attack and computer virus. The weights of disconnected links, indicating the workload of the links, can be transferred or redistributed to newly connected links to maintain network operations. Distinctively different from existing studies focused on adaptive unweighted networks, this paper presents a new mean-field model to analyze the reliability of adaptive weighted networks against cascading failures. By taking mean-field approximation, we develop a new continuous-time Markov model to capture the propagations of cascading failures, and the rewiring actions that individual nodes can take to bypass failed neighbors. We analyze the stability of the model to identify the critical conditions, under which the cascading failures can be eventually inhibited or would proliferate. The conditions are evaluated under different link weight distributions and rewiring strategies. Our model reveals that preferentially disconnecting suspicious peers with high weights can effectively inhibit virus and failures.
\end{abstract}

Index Terms-Adaptive weighted network, rewiring strategy, reliability.

\section{INTRODUCTION}

A LLOWING nodes to adaptively connect to reliable neighbors and disconnect those unreliable, a self-healing adaptive network is able to operate based on the credibility and reliability of individual nodes, and inhibit virus spread and cascading failures. Adaptive (weighted) networks have become increasingly important, as a result of the proliferation of the cloud computing [1-3], vehicular ad-hoc networks (VANETs) [4], and social networks [5-7]. Adaptive (weighted) networks are of particular interest in practice, where attacks

This work was supported by the National Natural Science Foundation of China (Nos. 61672298, 61873326, 61373136, 61802155), the Philosophy Social Science Research Key Project Fund of Jiangsu University (No. 2018SJZDI142) and the Research Foundation for Humanities and Social Sciences of Ministry of Education of China (Nos. 17YJAZH071).

B. Song is with the college of Telecommunication \& Information Engineering, Nanjing University of Posts and Telecommunications, Nanjing, China, 210003 (e-mail: songbo19870510@126.com).

$\mathrm{X}$. Wang is with the School of Cyberspace Security, Beijing University of Posts and Telecommunications, Beijing, China, 100876 (e-mail: xu.wang1@uts.edu.au).

B. Song and X. Wang are also with the Global Big Data Technologies Centre, University of Technology Sydney, Australia, 2007.

W. $\mathrm{Ni}$ is with CSIRO, Sydney, Australia, NSW 2122 (e-mail: wei.ni@data61.csiro.au).

Y. R. Song and G. P. Jiang are with the college of Automation, Nanjing University of Posts and Telecommunications, and Jiangsu Engineering Lab for IOT Intelligent Robots (IOTRobot), Nanjing, China, 210023. (e-mail: songyr@njupt.edu.cn and jianggp@njupt.edu.cn).

R. P. Liu and Y. J. Guo are with the Global Big Data Technologies Centre, University of Technology Sydney, Ultimo, NSW 2007, Australia (e-mail: renping.liu@uts.edu.au and Jay.Guo@uts.edu.au). are often strategic and responsive to defenders' actions. The networks can combat strategic attacks [8], by rewiring to bypass attacked nodes $[5-7,9,10]$. As a result, the topology of the network keeps changing in response to the attacks, confusing the attackers, counteracting strategic attacks (e.g., to strategically critical nodes with high degrees in static networks), and transferring the strategic attacks to exhibit stationarity.

An example of adaptive weighted networks is network function virtualization (NFV) on cloud computing platforms, where a large number of virtual machines (VMs) are installed, running virtual network functions (VNFs) [11-13]. The VMs are connected through virtual links. Network services need to be processed at different VMs running different VNFs in correct orders. The VMs and virtual links can be configured in response to requests of network services, and the weight of a virtual link can indicate the workload of services that a VM partially completes and forwards to another VM for further processing. In the case where some VMs are congested due to distributed denial-of-service (DDoS) attacks or infected due to computer viruses, new virtual links can be established to bypass these VMs. The weights (or in other words, the workloads) of the disconnected virtual links can be transferred to the new links. The VMs that are neither attacked or infected can check their routing tables, decide to rewire their virtual links. The number of the new connections can be set to be equal to the number of links disconnected, so as to maintain the consistency of workload execution and the controllability of NFV.

Studies have been carried out to design rewiring protocols and analyze rewiring effects, typically in adaptive unweighted networks $[6,7,9,14]$, where rewiring is random and independent of the logical or geographical closeness between a specific pair of nodes. In practice, there are great potentials for a healthy node to disconnects suspicious neighbors based on the frequency of communication occurrences. A healthy node may preferentially disconnect a frequently communicated, suspicious neighbor, so as to prevent cascading failures, such as DDoS attacks and virus infection, in NFV. Alternatively, a healthy node may choose to disconnect infrequently communicated, suspicious neighbors, so as to maintain the functionality of the network for intensive urgent tasks at the cost of network failures in the long term.

The conditions inhibiting and facilitating virus spread or cascading failures are important to the analysis of network reliability. Extensive studies have been carried out on the conditions in conventional networks without rewiring or weighting 
of network links, by using the susceptible-infected-susceptible (SIS) models [15-17]. To the best of our knowledge, however, there has been no rigorous analytical study on the emerging adaptive weighted networks [18, 19]. A key challenge is that not only can the nodes change states (as modeled in typical SIS models [16]), but the links connecting the nodes can also rewire and change over time (as opposed to the typical SIS models). Another critical challenge is that the links can be differently weighted. These challenges cannot be straightforwardly addressed by existing SIS models. Nontrivial extensions of the models are required.

This paper presents a new mean-field model to analyze the resistance of adaptive weighted networks against cascading failures, such as DDoS attack and computer virus. As a consequence of the new challenges, new derivations are necessary to extend the SIS model and evaluate the impact of rewiring and of weighted network links on the reliability of the adaptive weighted networks:

1) A new set of differential equations are formulated to model the continuous-time Markov chain process of the rewiring of weighted links in adaptive weighted networks. The differential equations are linearized. The largest eigenvalue of the Jacobian matrix of the linearization is the key to the study of the network reliability, but is not readily achievable.

2) We judiciously decompose the Jacobian matrix, evaluate the eigenvalues of the different parts by using determinant transformations and spectral analysis, and finally unveil the range of the largest eigenvalue of the Jacobian matrix. The upper and lower bounds of the range provide the sufficient conditions for the inhibition and proliferation of virus or cascading failures in adaptive weighted networks.

3) Two case studies verify the conditions, with exponentially and log-normally distributed link weights. By exploiting Order Statistics and Taylor expansion, we reveal that the condition of proliferation of virus or cascading failures is inversely proportional to both the network degree and average link weight.

Extensive simulations confirm the validity of the identified conditions, as well as the effectiveness of adaptive weighted networks in terms of suppressing cascading failures. An important finding is that the distributions of the link weights can have a strong impact on network reliability against virus spread or cascading failures in adaptive weighted networks. This is distinctively different from the existing conclusions on current static weighted networks [17]. We also find that the higher upper bound the rewiring rates of the weighted network links have, the more robust the adaptive weighted networks are against outbreaks of virus or failures. In the case of nonuniform rewiring rates, the distributions of the rewiring rates can also have a marked impact on the network reliability.

The rest of this paper is organized as follows. In Section II, the related works are reviewed. In Section III, the structure of adaptive weighted network is described. The proposed meanfield model of adaptive weighted network is presented in Section IV, followed by the stability analysis of the adaptive weighted networks. Two rewiring strategies are discussed and evaluated in Section VI. In Section VII, numerical and simulation results are provided, followed by conclusions in Section VIII.

\section{RELATED WORK}

In [9], an adaptive unweighted network was first proposed to describe interactions between a time-varying network topology, as well as the dynamics of the nodes that emerges when an infectious disease spreads on a social network. An SIS epidemic model was developed, where a susceptible node could break its links with its infected neighbors with a fixed probability and reconnect to other randomly selected susceptible nodes. It was found that this rewiring process can significantly increase the threshold for the epidemic breakout. Later, the interaction between epidemic processes and the network topology has been extensively studied under the assumption of unweighted links [7, 10, 14]. Particularly, Song et al. [20] proposed a new preferentially reconnecting edge strategy depending on spatial distance (PR-SD), where a new link is established at random with probability $p$ and in the shortest distance with the probability $(1-p)$.

By using Cellular Automata [21], an epidemic model was designed for unweighted adaptive networks, and the effectiveness of the model was demonstrated by numerical simulations. Yang et al. [14] found that a strong community structure could result from rewiring at the early stage of epidemic spread, based on the adaptive SIS model of [9]. Two community-based rewiring control strategies were proposed with a counterintuitive conclusion discovered: even the implementation of control measures unnecessarily result in the prevention of epidemic proliferation. Ilker et al. [22] proposed that healthy individuals choose to deactivate their contacts with infected neighbors, and only reactivate after the neighbors recover. A mean-field description of this system was developed with two distinct regimes identified: $(a)$ slow network dynamics, where the effective number of contacts per individual is reduced; and $(b)$ fast network dynamics, where the spread of disease is prevented by targeting suspicious connections. Demirel et al. [23] studied the dynamics of epidemic diseases in an adaptive unweighted network where nodes can be removed due to disease-induced mortality. Leonhard et al. [24] studied structural changes of adaptive unweighted networks and analyzed the interplay between topology and node-state dynamics near criticality. Zhu et al. [25] studied the epidemic spread process inside an adaptive unweighted network by taking into account that subjects could take preventive measure: cutting off connections with potential infection sources. Sherborne et al. [26] proposed a simple SIS epidemic model to study the time-delay rewiring in an unweighted network.

On the other hand, existing research has increasingly shown that the links between nodes are weighted, e.g., in traffic network, computer network and social network, and the distribution of link weights has a strong impact on epidemic behavior (even in static networks). Wang et al. [27] developed an edge-weight-based compartmental approach to study epidemic spread on networks with general degree and 
weight distributions, and drew the conclusion that the increasing heterogeneity of link weights can help suppress the epidemic spread. Zha et. al [4] employed probabilistic key predistribution to speed up message authentications, reduce communication costs, and support opportunistic routing under fast-changing topologies of distributed mobile networks. By using 3D Markov models, link-level analysis was carried out to evaluate the interaction between authentication success and radio success rate. Yan et al. [28] investigated epidemic spread in scale-free networks [29] with link weights indicating familiarity or closeness between two individuals. Numerical studies showed that large dispersions of the link weights can slow down the epidemic spread.

Taking into account the fact that the contact strengths among individuals are diverse, adaptive weighted networks have been increasingly interesting [18, 19, 30, 31]. Zhou et al. [18] numerically simulated the adaptive weighted networks and found that the weight adaption process could aggravate the prevalence of an epidemic, and become detrimental. Feng et al. [31] considered epidemic spread over an adaptive weighted network, in which the network topology varies according to the global and local infective information of individuals. Interacting strength is defined to evaluate the level of how individuals infective information takes effect on their connections. Discrete-time Monte-Carlo simulations were conducted with an initial $B A$ scale-free network. It was found that greater interacting strengths lead to higher epidemic thresholds, lower average disease densities of steady-state and shorter epidemic prevalent decay durations. Sun et al. [32] studied the spread of epidemic diseases in adaptively weighted scale-free networks. $\mathrm{Hu}$ et al. [33] changed the weights of links in an adaptive weighted network to balance the trade-off between the overall infection level and individual weight adaptation cost. Individuals could adapt their contact strengths to inhibit epidemic spread. No rewiring was considered in these works except [19] where only a numerical study was carried out.

Despite the adaptive weighted networks have been increasingly studied in different contexts of epidemics [19, 32], social network [31, 33], and computer network [18], a rigorous analysis of reliability of the networks capable of rewiring is yet to be delivered in the literature. The impact of rewiring weighted links on the reliability of the networks has not been understood. To the best of our knowledge, the only existing attempt to model the adaptive weighted networks capable of rewiring is in [19], where numerical simulations were heavily relied on to evaluate the network reliability. In contrast to the existing studies, this paper models analytically the rewiring of weighted links, and derives critical conditions of the rewiring rate under which virus or cascading failures can be inhibited or become insuppressible.

\section{AdAptive Weighted Network Structure}

Consider a generic network of $N$ nodes connected by $L$ weighted bidirectional links. The weights of the links are collected in $\boldsymbol{W}=\left\{w_{1}, w_{2}, \cdots, w_{M}\right\}, w_{i}>0, i=1,2, \cdots, M$, where $M$ is the number of different weights, measuring the closeness between two connected nodes (e.g., in terms of distance or communication frequency). The higher a weight is, the closer two connected nodes are. The probability distribution of $w_{i}$ is denoted by $g\left(w_{i}\right)$.

With reference to the SIS epidemic models [34], each node of the network can be in either a healthy/susceptible (S) or unhealthy/infected (I) state, indicating the node is reliable or not, respectively. We assume stationary random infections or failures which are reasonable in adaptive weighted networks, as discussed in Section I. Moreover, the assumption of stationary random infections has been extensively assumed in the existing SIS models, even in the case where the networks are static and could be vulnerable to strategic attacks, such as $[5,7,10,16,17]$. At any instant, for a $w$-weighted link connecting an unreliable node and a reliable node (SI/IS link), the reliable node can become unreliable with the rate $\beta_{w}=\tau w$ [17]. $\tau$ is a coefficient known in prior. The unreliable node can recover with rate $\gamma$ as the result of patching or antivirus software updating.

We consider that the reliable nodes can protect themselves by disconnecting from unreliable neighbors and reconnecting to other reliable nodes, thereby preserving network reliability. With probability $r_{w}$ for an SI link weighted $w$, the reliable node breaks the link to the unreliable one and forms a new link to another randomly selected reliable node. The rewiring rate $r_{w}$ is a random variable in general cases. The weights of the disconnected links can be transferred to the new links, while the weights of other links remain unchanged. $r_{w}$ can depend on $w$, e.g., the closeness of the nodes. In the case of NFV, the weight of a virtual link can indicate the workload from one VM to another, as described in Section I. The virtual links to the congested/failed VMs can be rewired to other VMs, and the weights (or workloads) of the links can be transferred to the new links. We assume that the number of links is fixed, as predominantly assumed in the literature on adaptive (un)weighted networks, e.g., [5-7, 9, 10]. In many cases, the assumption is reasonable and practical to maintain the connectivity and controllability of the networks. In a special case where the entire network becomes alert to threats (e.g., known virus or failures), the rewiring rate can be independent of the link weight, i.e., $r_{w}=r, \forall w$ [16].

Fig. 1 presents the operations of a node in an adaptive weighted network, where DDoS attacks or computer viruses can propagate to explore vulnerabilities in the network [7]. The weight of the link between a pair of nodes can account for the frequency the nodes interact; or in other words, the workload the nodes send to each other. A susceptible (or healthy) node is more likely to be infected by an infected neighbor it interacts frequently, than by one it interacts infrequently. Once one of its neighbors is infected or fails, the node can observe the misbehaviors of the neighbor and rewire its link to bypass the infected neighbor, thereby preventing propagation of the attacks or failures [5-7]. As a result, the topology of the network keeps changing in response to attacks or failures, quarantining infected individuals and counteracting the vulnerability explorations.

Other notations are defined as follows: $[A](A \in\{S, I\})$ denotes the number of nodes in state $A$, and $[S]+[I]=N$. $[A B]_{w}$ denotes the number of edges weighted $w$, connecting two 
For a healthy node, and any of its active links weighted $w$

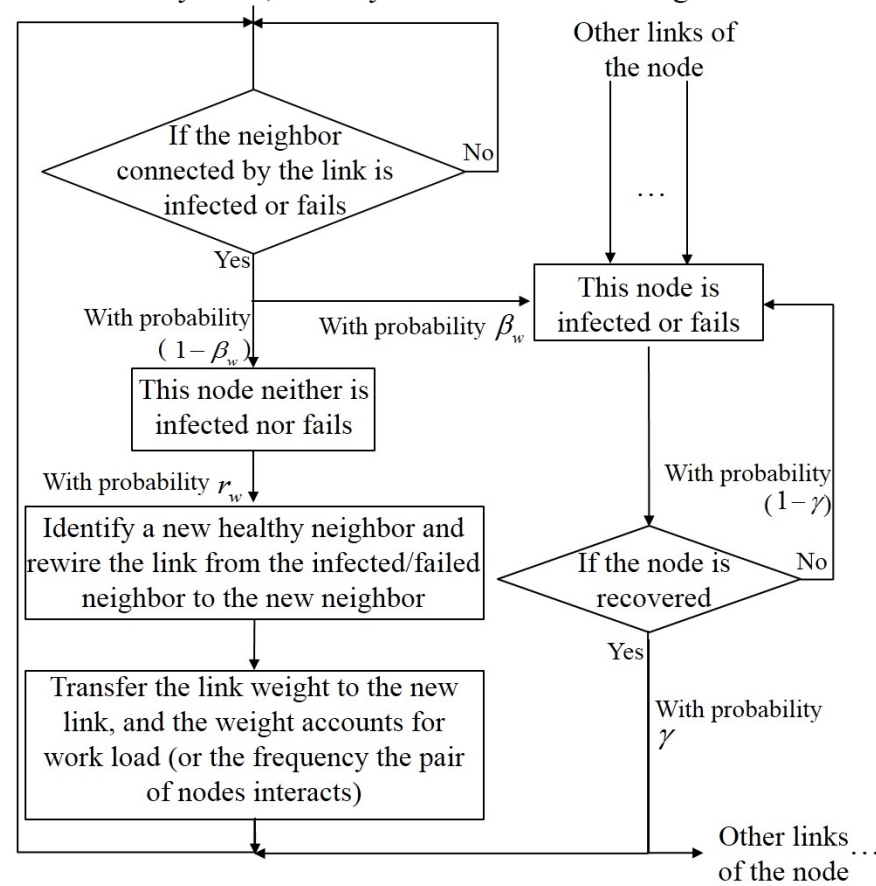

Fig. 1. The flowchart of a node in regards of a $w$-weighted link. The model is continuous-time and therefore the flowchart runs continuously. The detection of misbehaved neighbors can be done by using anti-virus features and techniques, e.g., [35], which are beyond the scope of this paper.

nodes in states $A$ and $B$, and $2[S I]_{w}+[S S]_{w}+[I I]_{w}=k N g(w)$. $k$ is the average degree of the network, and $2[S I]_{w}=[S I]_{w}+$ $[I S]_{w} . k[A]=[A A]+[A B]=\sum_{i=1}^{M}[A A]_{w_{i}}+\sum_{i=1}^{M}[A B]_{w_{i}}$. $[A B C]_{w w^{\prime}}$ denotes the number of triplets $A-B-C$, with edge $A B$ weighted $w$ and edge $B C$ weighted $w^{\prime}$.

\section{Proposed Mean-Field Model of Adaptive WEIGHTED NETWORK}

The research approach we take is to first model a new continuous-time Markov chain process to capture the rewiring and weighting of network links in adaptive weighted networks, and then analyze the conditions of the equilibriums of the model. The equilibriums considered in this paper are: (1) a disease-free equilibrium in which virus infections or cascading failures are completely eliminated; and (2) an outbreak equilibrium in which the infections or failures are insuppressible. In other words, the whole adaptive weighted network stabilize, either free of infections/failures, or with insuppressible infections/failures. By applying the HartmanGrobman theorem [36], the conditions of the equilibriums are analyzed by linearizing the model and evaluating the largest eigenvalue of the Jacobian matrix of the linearization. With mathematical manipulation, we derive the upper and lower bounds of the largest eigenvalue, which provide the sufficient conditions respectively for the proliferation and inhibition of virus or cascading failures in adaptive weighted networks.

Mean-field approximations are taken to improve the tractability of the continuous-time Markov Chain process. Mean-field theory studies the behavior of large and complex stochastic models where a large number of small individual components can interact with each other [15]. The mean-field approximations use a single average effect to approximate the effect of all the other individuals on any given individual. As a result, the interactions between individuals can be decoupled for analytical tractability, and the populations of individuals with different characteristics can be studied. The mean-field approximation is suitable for large-scale networks [37].

The time-varying populations of the nodes and the links are captured by a set of differential equations, as given by (1).

$$
\begin{aligned}
\frac{\mathrm{d}[S]}{\mathrm{d} t} & =\gamma \sum_{i}\left[I_{i}\right]-\sum_{i} \sum_{w} \beta_{w}\left[S_{i} I\right]_{w} ; \\
\frac{\mathrm{d}[I]}{\mathrm{d} t} & =-\gamma \sum_{i}\left[I_{i}\right]+\sum_{i} \sum_{w} \beta_{w}\left[S_{i} I\right]_{w} ; \\
\frac{\mathrm{d}[S S]_{w}}{\mathrm{~d} t} & =\gamma \sum_{i} \sum_{j}\left(\left[S_{i} I_{j}\right]_{w}+\left[I_{i} S_{j}\right]_{w}\right) \\
& +r_{w} \sum_{i} \sum_{j}\left(\left[S_{i} I_{j}\right]_{w}+\left[I_{i} S_{j}\right]_{w}\right) \\
& -\sum_{w^{\prime}} \beta_{w^{\prime}} \sum_{i} \sum_{j}\left(\left[S_{i} S_{j} I\right]_{w w^{\prime}}+\left[I S_{i} S_{j}\right]_{w^{\prime} w}\right) ; \\
\frac{\mathrm{d}[I I]_{w}}{\mathrm{~d} t} & =-2 \gamma \sum_{i} \sum_{j}\left[I_{i} I_{j}\right]_{w}+\beta_{w} \sum_{i} \sum_{j}\left(\left[S_{i} I_{j}\right]_{w}+\left[I_{i} S_{j}\right]_{w}\right) \\
& +\sum_{w^{\prime}} \beta_{w^{\prime}} \sum_{i} \sum_{j}\left(\left[I_{i} S_{j} I\right]_{w w^{\prime}}+\left[I S_{i} I_{j}\right]_{w^{\prime} w}\right) ; \\
\frac{\mathrm{d}[S I]_{w}}{\mathrm{~d} t} & =-\gamma \sum_{i} \sum_{j}\left(\left[S_{i} I_{j}\right]_{w}-\left[I_{i} I_{j}\right]_{w}\right)-\beta_{w} \sum_{i} \sum_{j}\left[S_{i} I_{j}\right]_{w} \\
& +\sum_{w^{\prime}} \beta_{w^{\prime}} \sum_{i} \sum_{j}\left(\left[S_{i} S_{j} I\right]_{w w^{\prime}}-\left[I S_{i} I_{j}\right]_{w^{\prime} w}\right) \\
& -r_{w} \sum_{i} \sum_{j}\left[S_{i} I_{j}\right]_{w} ; \\
\frac{\mathrm{d}[I S]_{w}}{\mathrm{~d} t} & =-\gamma \sum_{i} \sum_{j}\left(\left[I_{i} S_{j}\right]_{w}-\left[I_{i} I_{j}\right]_{w}\right)-\beta_{w} \sum_{i} \sum_{j}\left[I_{i} S_{j}\right]_{w} \\
& +\sum_{w^{\prime}} \beta_{w^{\prime}} \sum_{i} \sum_{j}\left(\left[I S_{i} S_{j}\right]_{w^{\prime} w}-\left[I_{i} S_{j} I\right]_{w w^{\prime}}\right) \\
& -r_{w} \sum_{i} \sum_{j}\left[I_{i} S_{j}\right]_{w}, \\
&
\end{aligned}
$$

where $\left[A_{i}\right]$ represents the probability that the node $i$ 's state is $A,\left[A_{i} B_{j}\right]$ represents the probability that a link connecting a pair of nodes in states $A$ and $B, \forall A, B \in\{S, I\}$. Here, (1a) captures the time-changing population of nodes in the healthy (or susceptible) state. The first term on the right-hand side (RHS) of (1a) corresponds to the part of the population recovering from the infected state with the probability of $\gamma$. The second term corresponds to the part of the population infected by their infected neighbors with the probability $\beta_{w}$. Likewise, (1b) captures the time-changing population of nodes in the infected state.

Eqs. (1c) and (1d) characterize the time-varying numbers of links weighted by different weights and connecting nodes in different states. For instance, (1c) captures the changing number of the $w$-weighted links connecting two healthy nodes. The first term on the RHS of (1c) is the increased part of 
the link number, resulting from the recovery of the infected ends of the links with the probability of $\gamma$. The second term is another increased part of the link number, resulting from rewiring to bypass an infected node with the probability of $r_{w}$. The third term is the number of the previous $w$-weighted $S S$ links which become $S I$ links due to the infection at one end of the links through a $w^{\prime}$-weighted link with the probability of $\beta_{w^{\prime}}$. Likewise, (1d) captures the time-changing number of $w$-weighted II links connecting a pair of infected nodes, (1e) and (1f) capture the time-changing number of $w$-weighted $S I$ and $I S$ links, respectively.

By taking the mean-field approximation, the expectation of infected nodes in the network can be written as the sum of the probability that each node in the network is infected, i.e., $[I]=\sum_{i}\left[I_{i}\right]=N\left[I_{i}\right]$. Similarly, the expectation of $w$-weighted $S I$ links can be written as $[S I]_{w}=\sum_{i} \sum_{j}\left[S_{i} I_{j}\right]_{w}$, by assuming all the $w$-weighted edges exhibit the same state. The temporal changes in the population of healthy nodes and infected nodes can be written as

$$
\begin{aligned}
& \frac{\mathrm{d}[S]}{\mathrm{d} t}=\gamma[\boldsymbol{I}]-\sum_{w} \beta_{w}[S I]_{w} \\
& \frac{\mathrm{d}[\boldsymbol{I}]}{\mathrm{d} t}=-\gamma[\boldsymbol{I}]+\sum_{w} \beta_{w}[S I]_{w},
\end{aligned}
$$

where (2a) captures the time-changing population of nodes in the healthy (or susceptible) state. The first term on the RHS of (2a) corresponds to the part of the population recovering from the infected state with the probability of $\gamma$. The second term corresponds to the part of the population infected by their infected neighbors with the probability of $\beta_{w}$. Likewise, (2b) captures the time-changing population of nodes in the infected state.

The temporal change of a link depends on its weight and the states of the nodes at both ends of the link. By taking the mean-field approximation, the expectation of the $w$-weighted $A B$ links in the network can be written as the sum of the probability that each link connecting a pair of $A$ node and $B$ node, i.e., $[A B]_{w}=\sum_{i} \sum_{j}\left[A_{i} B_{j}\right]_{w}, \forall A, B \in\{S, I\}$. The temporal changes in the numbers of links of different types can be written as

$$
\begin{aligned}
\frac{\mathrm{d}[S S]_{w}}{\mathrm{~d} t} & =\gamma\left([S I]_{w}+[I S]_{w}\right)+r_{w}\left([S I]_{w}+[I S]_{w}\right) \\
& -\sum_{w^{\prime}} \beta_{w^{\prime}}\left([S S I]_{w w^{\prime}}+[I S S]_{w^{\prime} w}\right) ; \\
\frac{\mathrm{d}[I I]_{w}}{\mathrm{~d} t} & =-2 \gamma[I I]_{w}+\beta_{w}\left([S I]_{w}+[I S]_{w}\right) \\
& +\sum_{w^{\prime}} \beta_{w^{\prime}}\left([I S I]_{w w^{\prime}}+[I S I]_{w^{\prime} w}\right) ; \\
\frac{\mathrm{d}[S I]_{w}}{\mathrm{~d} t} & =-\gamma[S I]_{w}+\gamma[I I]_{w}-\beta_{w}[S I]_{w} \\
& +\sum_{w^{\prime}} \beta_{w^{\prime}}\left([S S I]_{w w^{\prime}}-[I S I]_{w^{\prime} w}\right)-r_{w}[S I]_{w} ; \\
\frac{\mathrm{d}[I S]_{w}}{\mathrm{~d} t} & =-\gamma[I S]_{w}+\gamma[I I]_{w}-\beta_{w}[I S]_{w} \\
& +\sum_{w^{\prime}} \beta_{w^{\prime}}\left([I S S]_{w^{\prime} w}-[I S I]_{w w^{\prime}}\right)-r_{w}[I S]_{w} .
\end{aligned}
$$

Eqs. (3a) and (3b) characterize the time-varying numbers of links weighted by different weights and connecting nodes in different states. For instance, (3a) captures the time-changing number of the $w$-weighted links connecting two healthy nodes. The first term on the RHS of (3a) results from the recovery of the infected ends of the links with the probability of $\gamma$. The second term on the RHS of (3a) results from rewiring to bypass an infected node with the probability of $r_{w}$. The third term is the number of previous $w$-weighted $S S$ links which become SI links due to the infection at one end of the links through a $w^{\prime}$-weighted link with the probability of $\beta_{w^{\prime}}$. Likewise, (3b) captures the time-changing number of $w$ weighted II links connecting a pair of infected nodes; and (3c) and (3d) capture the time-changing number of $w$-weighted $S I$ and $I S$ links, respectively.

We assume that the weight of a link is symmetry, i.e., $w(i, j)=w(j, i), \forall i \neq j$. Then $[S I]_{w}=[I S]_{w}$. (2) and (3) can be rewritten as

$$
\begin{aligned}
\frac{\mathrm{d}[S]}{\mathrm{d} t} & =\gamma[I]-\sum_{w} \beta_{w}[S I]_{w} \\
\frac{\mathrm{d}[I]}{\mathrm{d} t} & =-\gamma[I]+\sum_{w} \beta_{w}[S I]_{w} \\
\frac{\mathrm{d}[S S]_{w}}{\mathrm{~d} t} & =2 \gamma[S I]_{w}-2 \sum_{w^{\prime}} \beta_{w^{\prime}}[S S I]_{w w^{\prime}}+2 r_{w}[S I]_{w} \\
\frac{\mathrm{d}[I I]_{w}}{\mathrm{~d} t} & =-2 \gamma[I I]_{w}+2 \beta_{w}[S I]_{w}+2 \sum_{w^{\prime}} \beta_{w^{\prime}}[I S I]_{w w^{\prime}} ; \\
\frac{\mathrm{d}[S I]_{w}}{\mathrm{~d} t} & =-\gamma[S I]_{w}+\gamma[I I]_{w}-\beta_{w}[S I]_{w} \\
& +\sum_{w^{\prime}} \beta_{w^{\prime}}\left([S S I]_{w w^{\prime}}-[I S I]_{w^{\prime} w}\right)-r_{w}[S I]_{w}
\end{aligned}
$$

which is the mean-field approximation of the continuous-time Markov chain model for the adaptive weighted networks. The time-varying states of both the nodes and links are captured.

We note that the linear expressions in (4) are due to the fact that the system of interest is continuous-time. At every time instant $\Delta t \rightarrow 0$, the probability of a healthy node being infected by more than one infected neighbor approaches zero.

We can apply the moment closure approximation [17] to evaluate $[A]$ and $[A B]_{w}$, and the number of triplets $[A B C]_{w w^{\prime}}$ can be written as [38]:

$$
[A B C]_{w w^{\prime}}=\xi \frac{[A B]_{w}[B C]_{w^{\prime}}}{[B]},
$$

where $\xi=\frac{k-1}{k}$. Based on (5), we can have

$$
\begin{aligned}
& {[S S I]_{w w^{\prime}}=\xi \frac{[S S]_{w}[S I]_{w^{\prime}}}{[S]},} \\
& {[I S I]_{w w^{\prime}}=\xi \frac{[S I]_{w}[S I]_{w^{\prime}}}{[S]} .}
\end{aligned}
$$

By substituting (6) and (7) into (4), we can rewrite (4) as

$$
\frac{\mathrm{d}[S]}{d t}=\gamma[I]-\sum_{w} \beta_{w}[S I]_{w}
$$




$$
\begin{aligned}
\frac{\mathrm{d}[I]}{d t}= & -\gamma[I]+\sum_{w} \beta_{w}[S I]_{w} \\
\frac{\mathrm{d}[S S]_{w}}{d t}= & 2\left(\gamma+r_{w}\right)[S I]_{w}-2 \xi \frac{[S S]_{w}}{[S]} \sum_{w^{\prime}} \beta_{w^{\prime}}[S I]_{w^{\prime}} \\
\frac{\mathrm{d}[I I]_{w}}{d t}= & -2\left(\gamma[I I]_{w}-\beta_{w}[S I]_{w}-\xi \frac{[S I]_{w}}{[S]} \sum_{w^{\prime}} \beta_{w^{\prime}}[S I]_{w^{\prime}}\right) \\
\frac{\mathrm{d}[S I]_{w}}{d t}= & -\left(\gamma+\beta_{w}+r_{w}\right)[S I]_{w}+\gamma[I I]_{w} \\
& +\xi \frac{[S S]_{w}-[S I]_{w}}{[S]} \sum_{w^{\prime}} \beta_{w^{\prime}}[S I]_{w^{\prime}}
\end{aligned}
$$

For the purpose of cross-validation of the proposed model, we consider a special case where the rewiring rate $r_{w}=r$ is a constant. When $r_{w}=0$, i.e., the network is static, and (8) can be rewritten in the exactly same way as [16, eq.6] describing static weighted networks. In other words, (8) is cross-validated by the special case.

\section{Stability Analysis of Adaptive Weighted NETWORK}

We proceed to derive the reliability threshold of $\tau$, denoted by $\tau^{*}$ based on (8). $\tau^{*}$ is the evaluation of the reliability of the adaptive weighted networks against cascading failures. If $\tau<\tau^{*}$, the adaptive weighted network can eventually become reliable, i.e., all nodes eventually become reliable. Otherwise, the network is unreliable, i.e., the unreliability proliferates. The larger $\tau^{*}$ is, the more resilient the network is, e.g., against virus spread and cascading failures. To derive $\tau^{*}$, we analyze the stability of the equilibrium of the adaptive weighted networks.

As stated in the Lyapunov's first method [39], the behavior of a dynamical system in a domain near an equilibrium point is qualitatively the same as the behavior of its linearization near this equilibrium point. If and only if the Jacobian matrix of the linearization has all negative eigenvalues, a nonlinear dynamical system is stable at the equilibrium. The equilibrium point of interest, also known as the disease-free equilibrium point, is $\left(\frac{d[I I]_{w}}{d t}, \frac{d[S I]_{w}}{d t}\right)=(0,0)$, at which all nodes are reliable [40]. By exploiting the Lyapunov's first method, we linearize (8) in the vicinity of the equilibrium, evaluate the eigenvalues of the linearization at the equilibrium, and study the condition under which the Jacobian matrix of the linear system has all negative eigenvalues [41]. As a result, we are able to establish the thresholds to preserve stability or undergo instability at the equilibrium.

Based on the aforementioned condition $[S]+[I]=N$, $2[S I]_{w}+[S S]_{w}+[I I]_{w}=k N g(w)$ and $k[I]=[S I]+[I I],(8 \mathrm{~d})$ and (8e) can be respectively rewritten as

$$
\begin{aligned}
\frac{\mathrm{d}[I I]_{w}}{\mathrm{~d} t} & =-2 \gamma[I I]_{w}+2 \beta_{w}[S I]_{w} \\
& +\frac{2(k-1)[S I]_{w}}{k N-[S I]-[I I]} \sum_{w^{\prime}} \beta_{w^{\prime}}[S I]_{w^{\prime}} \\
\frac{\mathrm{d}[S I]_{w}}{d t} & =(k-1) \frac{k N g(w)-3[S I]_{w}-[I I]_{w}}{k N-[S I]-[I I]} \sum_{w^{\prime}} \beta_{w^{\prime}}[S I]_{w^{\prime}} \\
& -\left(\gamma+\beta_{w}+r_{w}\right)[S I]_{w}+\gamma[I I]_{w}
\end{aligned}
$$

By suppressing all higher order terms of $[I I]_{w}$ and $[S I]_{w},(9)$ can be linearized, as given by

$$
\begin{aligned}
\frac{\mathrm{d}[I I]_{w}}{\mathrm{~d} t} & \approx-2 \gamma[I I]_{w}+2 \beta_{w}[S I]_{w} \\
\frac{\mathrm{d}[S I]_{w}}{\mathrm{~d} t} & \approx-\left(\gamma+\beta_{w}+r_{w}\right)[S I]_{w}+\gamma[I I]_{w} \\
& +(k-1) g(w) \sum_{w^{\prime}} \beta_{w^{\prime}}[S I]_{w^{\prime}}
\end{aligned}
$$

The linear stability analysis of (10) is carried out in the vicinity of the equilibrium. By the Hartman-Grobman theorem [36], the behavior of the system around an equilibrium point can be evaluated through the eigenvalues of the Jacobian matrix of (10). Let $\mathbf{J}=\left[J_{i j}\right]$ denote the Jacobian matrix of (10) at the equilibrium, as given by [16]

$$
\mathbf{J}=\left(\begin{array}{ll}
\mathbf{J}^{11} & \mathbf{J}^{12} \\
\mathbf{J}^{21} & \mathbf{J}^{22}
\end{array}\right)
$$

where

$$
\begin{gathered}
\mathbf{J}^{11}=\operatorname{diag}[-2 \gamma,-2 \gamma, \cdots,-2 \gamma] ; \\
\mathbf{J}^{12}=\operatorname{diag}\left[2 \beta_{w_{1}}, 2 \beta_{w_{2}}, \cdots, 2 \beta_{w_{M}}\right] ; \\
\mathbf{J}^{21}=\operatorname{diag}[\gamma, \gamma, \cdots, \gamma] ; \\
J_{i j}^{22}= \begin{cases}(k-1) g\left(w_{i}\right) \beta_{w_{j}}, & \text { if } i \neq j ; \\
-\left(\gamma+\beta_{w_{i}}+r_{w_{i}}\right)+(k-1) g\left(w_{i}\right) \beta_{w_{i}}, & \text { if } i=j .\end{cases}
\end{gathered}
$$

Note that the matrix block $\mathbf{J}^{11}$ is an $M$-by- $M$ diagonal matrix. By block matrix multiplication, we can get

$$
\left(\begin{array}{ll}
\mathbf{J}^{11} & \mathbf{J}^{12} \\
\mathbf{J}^{21} & \mathbf{J}^{22}
\end{array}\right)\left(\begin{array}{cc}
\mathbf{I}_{M} & -\mathbf{J}^{11^{-1}} \mathbf{J}^{12} \\
0 & \mathbf{I}_{M}
\end{array}\right)=\left(\begin{array}{cc}
\mathbf{J}^{11} & 0 \\
\mathbf{J}^{21} & \mathbf{H}
\end{array}\right)
$$

where $\mathbf{I}_{M}$ is the identity matrix, and $\mathbf{H}=\mathbf{J}^{22}-\mathbf{J}^{21} \mathbf{J}^{11^{-1}} \mathbf{J}^{12}$.

Let row vector $\boldsymbol{\mu}$ be the spectrum of the square matrix $\mathbf{J}$, i.e., collects all eigenvalues of $\mathbf{J}$, and $\mu_{i} \in \boldsymbol{\mu}$ be the $i$-th (largest) eigenvalue of $\mathbf{J}, i=1,2, \cdots, 2 M$. The characteristic polynomial of $\mathbf{J}$ can be written as

$$
\begin{aligned}
\operatorname{det}\left[\mathbf{J}-\mu_{i} \mathbf{I}_{2 M}\right] & =\operatorname{det}\left[\left(\begin{array}{cc}
\mathbf{J}^{11} & 0 \\
\mathbf{J}^{21} & \mathbf{H}
\end{array}\right)-\mu_{i} \mathbf{I}_{2 M}\right] \\
& =\operatorname{det}\left[\begin{array}{cc}
\mathbf{J}^{11}-\lambda_{i} \mathbf{I}_{M} & 0 \\
\mathbf{J}^{21} & \mathbf{H}-\eta_{i} \mathbf{I}_{M}
\end{array}\right],
\end{aligned}
$$

where $\lambda_{i}$ and $\eta_{i}$ are the $i$-th eigenvalues of $\boldsymbol{\lambda}$ and $\boldsymbol{\eta}$, i.e., the spectra of the square matrices $\mathbf{J}^{\mathbf{1 1}}$ and $\mathbf{H}$, respectively.

Then we have $\operatorname{det}\left[\mathbf{J}-\mu_{i} \mathbf{I}_{2 M}\right]=0$, so that

$$
\operatorname{det}\left[\mathbf{J}^{11}-\lambda_{i} \mathbf{I}_{M}\right]=0 ; \operatorname{det}\left[\mathbf{H}-\eta_{i} \mathbf{I}_{M}\right]=0 ;
$$

in other words, $[\boldsymbol{\lambda}, \boldsymbol{\eta}]$ is also the spectrum of $\mathbf{J}$ from (13).

In [41], the linear state model (10) is stable at the equilibrium, if and only if the real parts of all the eigenvalues of $\mathbf{J}$ are negative. Since $\mathbf{J}^{11}$ is an $M$-by- $M$ diagonal matrix with all the main diagonal entries equal to $-2 \gamma$, all of the $M$ eigenvalues of $\mathbf{J}^{11}$ are $-2 \gamma<0$. To this end, we can have $\max \{\boldsymbol{\mu}\}<0$, if and only if the maximum eigenvalue of $\mathbf{H}$, denoted by $\eta_{\max }(\mathbf{H})<0$. Let $\mathbf{H}=\left[H_{i j}\right]$, we have

$$
H_{i j}= \begin{cases}(k-1) g\left(w_{i}\right) \beta_{w_{j}}, & \text { if } i \neq j ; \\ -\left(\gamma+r_{w_{i}}\right)+(k-1) g\left(w_{i}\right) \beta_{w_{i}}, & \text { if } i=j .\end{cases}
$$


To evaluate $\eta_{\max }(\mathbf{H})$, we decouple $\mathbf{H}$ as $\mathbf{H}=\mathbf{H}^{(1)}+\mathbf{H}^{(2)}$, and rewrite $\mathbf{H}^{(1)}=\left[H_{i j}^{(1)}\right]$ and $\mathbf{H}^{(2)}=\left[H_{i j}^{(2)}\right]$. Then,

$$
H_{i j}=H_{i j}^{(1)}+H_{i j}^{(2)}
$$

where,

$$
H_{i j}^{(1)}= \begin{cases}(k-1) g\left(w_{i}\right) \beta_{w_{j}}, & \text { if } i \neq j \\ -\gamma+(k-1) g\left(w_{i}\right) \beta_{w_{i}}, & \text { if } i=j,\end{cases}
$$

and

$$
H_{i j}^{(2)}= \begin{cases}0, & \text { if } i \neq j \\ -r_{w_{i}}, & \text { if } i=j .\end{cases}
$$

By substituting (15), we can write $\operatorname{det}\left[\mathbf{H}^{(1)}\right]$, as given by

$$
(k-1)^{M}\left(\prod_{i=1}^{M} g\left(w_{i}\right) \beta_{w_{i}}\right)\left|\begin{array}{ccc}
1+x_{1} & \cdots & 1 \\
1 & \cdots & 1 \\
\vdots & \ddots & \vdots \\
1 & \cdots & 1+x_{M}
\end{array}\right|,
$$

where for notational simplicity, we define

$$
x_{i}=-\frac{\gamma}{(k-1) g\left(w_{i}\right) \beta_{w_{i}}}, i=1,2, \cdots, M .
$$

According to basic determinant transformations, we can have

$$
\begin{aligned}
& \left|\begin{array}{cccc}
1+x_{1} & 1 & \cdots & 1 \\
1 & 1+x_{2} & \cdots & 1 \\
\vdots & \vdots & \ddots & \vdots \\
1 & 1 & \cdots & 1+x_{M}
\end{array}\right| \\
& \stackrel{(a)}{=}\left|\begin{array}{cccc}
1+x_{1} & 1 & \cdots & 1 \\
-x_{1} & x_{2} & \cdots & 0 \\
\vdots & \vdots & \ddots & \vdots \\
-x_{1} & 0 & \cdots & x_{M}
\end{array}\right| \\
& =\prod_{i=1}^{M} x_{i}\left|\begin{array}{cccc}
1+\frac{1}{x_{1}} & \frac{1}{x_{2}} & \cdots & \frac{1}{x_{M}} \\
-1 & 1 & \cdots & 0 \\
\vdots & \vdots & \ddots & \vdots \\
-1 & 0 & \cdots & 1
\end{array}\right| \\
& \stackrel{(b)}{=} \prod_{i=1}^{M} x_{i}\left|\begin{array}{ccccc}
1+\sum_{i=1}^{M} \frac{1}{x_{i}} & \frac{1}{x_{2}} & \cdots & \frac{1}{x_{M}} \\
0 & 1 & \cdots & 0 \\
\vdots & \vdots & \ddots & \vdots \\
0 & 0 & \cdots & 1
\end{array}\right| \\
& \stackrel{(c)}{=}\left(\prod_{i=1}^{M} x_{i}\right)\left(1+\sum_{i=1}^{M} \frac{1}{x_{i}}\right),
\end{aligned}
$$

where $(a)$ is achieved by subtracting the first row from all the rest of rows; $(b)$ is achieved by adding all the other columns to the first column; $(c)$ is obtained by multiplying the elements of the first column to their respective minors.

As a result, (17) can be rewritten as $\operatorname{det}\left[\mathbf{H}^{(1)}\right]$

$=(k-1)^{M}\left(\prod_{i=1}^{M} g\left(w_{i}\right)\right)\left(\prod_{i=1}^{M} \beta_{w_{i}}\right)\left(\prod_{i=1}^{M} x_{i}\right)\left(1+\sum_{i=1}^{M} \frac{1}{x_{i}}\right)$ $=(-\gamma)^{M-1}\left(-\gamma+(k-1)\left\langle\beta_{w}\right\rangle\right)$, where $\left\langle\beta_{w}\right\rangle=\sum_{n=1}^{M} g\left(w_{n}\right) \beta_{w_{n}}$ takes the expectation of $\beta_{w}$.

The spectrum of $\mathbf{H}^{(1)}$, denoted accordingly by $\boldsymbol{\eta}^{(1)}$, satisfies

$$
\operatorname{det}\left[\mathbf{H}^{(1)}-\eta_{i}^{(1)} \mathbf{I}\right]=0 .
$$

According to (19), we have

$$
\left(-\gamma-\eta_{i}^{(1)}\right)^{M-1}\left(-\gamma+(k-1)\left\langle\beta_{w}\right\rangle-\eta_{\max }^{(1)}\right)=0 .
$$

By solving (20), one can obtain

$$
\begin{gathered}
\eta_{i}^{(1)}=-\gamma, i=2, \cdots, M ; \\
\eta_{1}^{(1)}=\eta_{\max }^{(1)}=(k-1)\left\langle\beta_{w}\right\rangle-\gamma,
\end{gathered}
$$

where $\eta_{\max }^{(1)}$ is the largest eigenvalue of $\mathbf{H}^{(1)}$ and $\eta_{i}^{(1)}$ is any other eigenvalue.

Let $\zeta=\gamma+\max \left\{r_{w_{i}}\right\}+1$, then $\mathbf{H}+\zeta \mathbf{I}>\mathbf{0}$, where all the entries are positive. Since $\mathbf{H}=\mathbf{H}^{(1)}+\mathbf{H}^{(2)}$ and $\mathbf{H}^{(2)}$ is a diagonal matrix, we can get

$$
\mathbf{0}<\mathbf{H}^{(1)}+\eta_{\min }^{(2)} \mathbf{I}+\zeta \mathbf{I} \leq \mathbf{H}+\zeta \mathbf{I} \leq \mathbf{H}^{(1)}+\eta_{\max }^{(2)} \mathbf{I}+\zeta \mathbf{I},
$$

where $\eta_{\min }^{(2)}=\min _{i}\left\{-r_{w_{i}}\right\}$ and $\eta_{\max }^{(2)}=\max _{i}\left\{-r_{w_{i}}\right\}$. Matrices $\mathbf{P} \leq \mathbf{Q}$ stands for that entry of $\mathbf{P}$ is no less than the corresponding entry of $\mathbf{Q}$.

By Perron-Frobenius theorem [42], for any matrices $\mathbf{A}$ and $\mathbf{B}$ with $0 \leq \mathbf{A} \leq \mathbf{B}$, the spectral radii of $\mathbf{A}$ and $\mathbf{B}$ satisfy $\rho(\mathbf{A}) \leq \rho(\mathbf{B})$. For $\mathbf{A}=\left[a_{i j}\right]$ with $a_{i j}>0, \forall i, j$, the spectral radius $\rho(\mathbf{A})$ is equal to the largest eigenvalue. Therefore, $\eta_{\max }(\mathbf{H})$ satisfies

$$
\eta_{1}^{(1)}+\eta_{\min }^{(2)}+\zeta \leq \eta_{\max }(\mathbf{H})+\zeta \leq \eta_{1}^{(1)}+\eta_{\max }^{(2)}+\zeta,
$$

i.e.,

$$
\eta_{1}^{(1)}+\eta_{\min }^{(2)} \leq \eta_{\max }(\mathbf{H}) \leq \eta_{1}^{(1)}+\eta_{\max }^{(2)} .
$$

From [41], the equilibrium is stable, if $\eta_{\max }(\mathbf{H}) \leq \eta_{1}^{(1)}+$ $\eta_{\max }^{(2)}<0$; and the equilibrium can be unstable, if $\eta_{\max }(\mathbf{H}) \geq$ $\eta_{1}^{(1)}+\eta_{\min }^{(2)}>0$. Since $\beta_{w}=\tau w,\left\langle\beta_{w}\right\rangle=\tau\langle w\rangle$, we have

$$
\begin{aligned}
& \eta_{1}^{(1)}+\eta_{\max }^{(2)}=(k-1) \tau\langle w\rangle-\gamma+\eta_{\max }^{(2)}, \\
& \eta_{1}^{(1)}+\eta_{\min }^{(2)}=(k-1) \tau\langle w\rangle-\gamma+\eta_{\min }^{(2)} .
\end{aligned}
$$

The network is reliable if

$$
\tau<\tau_{l}^{*}=\frac{\gamma+\min _{i}\left\{r_{w_{i}}\right\}}{(k-1)\langle w\rangle},
$$

where $\tau_{l}^{*}$ gives the lower bound of $\tau$ in reliable states.

The network is unreliable if $\eta_{\max }(\mathbf{H}) \geq \eta_{1}^{(1)}+\eta_{\min }^{(2)}>0$, then we have

$$
\tau>\tau_{u}^{*}=\frac{\gamma+\max _{i}\left\{r_{w_{i}}\right\}}{(k-1)\langle w\rangle},
$$

where $\tau_{u}^{*}$ gives the upper bound of $\tau$ in unreliable states.

In the special case where $r_{w_{i}}=r$, we have $\min _{i}\left\{r_{w_{i}}\right\}=$ $\max \left\{r_{w_{i}}\right\}=r$. Then, $\eta_{\max }(\mathbf{H})=(k-1)\left\langle\beta_{w}\right\rangle-^{i} \gamma-r$, and $\tau^{*}$ can be written explicitly in a closed-form, as given by

$$
\tau^{*}=\frac{\gamma+r}{(k-1)\langle w\rangle} .
$$


We can see in (24) that $\tau^{*}$ increases with the growth of the rewiring rate $r$, and decreases with the growth of the average link weights $\langle w\rangle$ in the case of uniform rewiring rates. It is also shown that the distribution of weight $w_{i}$ has little impact on $\tau^{*}$ when $r_{w_{i}}=r$. Moreover, we can see in (23) that, with non-uniform rewiring rates, the bounds of $\tau^{*}$ depend on $r_{w_{i}}$ which, in turn, depends on $w_{i}$. As the network becomes unreliable when $\tau>\tau^{*}$, we conclude that the higher the upper bound of $r_{w_{i}}$ is, the more resistant the network is against the outbreak. To this end, in the case of non-uniform rewiring rates $r_{w_{i}}$, the distribution of $w_{i}$ can have a strong impact on the upper bound of $r_{w_{i}}$ and thus the resistance of the network.

We note that our analysis is distinctively different from the existing studies. As discussed in Section II, the existing study of adaptive weighted networks, i.e., [19], is based on numerical evaluations and provides no analysis of the reliability of the networks. In different yet relevant contexts of (static) weighted networks and adaptive unweighted networks, analyses do avail, e.g., in [16] and [23], and may also evaluate the reliability by assessing the eigenvalues of the Jacobian. However, the analysis of weighted networks, e.g., [16], does not capture the rewiring rate $r_{w}$ which is key to the reliability threshold of adaptive weighted networks $\tau^{*}$; see (22) and (23). The analysis of adaptive unweighted networks, e.g., [23], cannot account for the non-uniform weights and the subsequent infection rates of adaptive weighted networks, which require new mathematic manipulations and lead to the new bounds of $\tau^{*}$. In contrast, we consider a new adaptive weighted network, where links can be rewired on-the-fly and the weights of disconnected links can be transferred to the new links. We develop a new continuous-time Markov model to characterize the changing states of the links, capturing the real-time rewiring process of the networks. With the non-trivial analysis of the Jacobian of the linearization of the model, the largest eigenvalue of the Jacobian is analyzed to specify the respective thresholds under which cascading failures can be inhibited or proliferate.

We also note that the link weights may not be symmetric in the presence of DDoS attacks. Our model can be readily applied to asymmetric link weights. As a matter of fact, (3) divides all the $w$-weighted links into four different types: $S S_{w}$, $I I_{w}, S I_{w}$ and $I S_{w}$, and provides the temporal changes in the numbers of links of the different types. $[I S]_{w}$ does not have to be equal to $[S I]_{w}$; or in other words, the link weights can be asymmetric. The above analysis, involving the linearization of differential equations, the derivation of the Jacobian of the linearized, and the evaluation of the eigenvalues of the Jacobian, can be readily based on (3).

\section{Rewiring Strategies and Network Stability}

As discussed in Section $\mathrm{V}$, the rewiring rate $r_{w_{i}}$ can be designed in different ways which can have a strong impact on the bounds of $\tau^{*}$. This section studies the impact by taking two different but simple linear designs of $r_{w_{i}}$ under two classical distributions of $w_{i}$ for example. Let $w_{(i)}$ denote the $i$-th smallest of $\boldsymbol{W} \in R^{M \times 1}$. Thus, $w_{(1)}=\min _{i}\left\{w_{i}\right\}$ and $w_{(M)}=\max _{i}\left\{w_{i}\right\}$.
The first design (Design 1) specifies the positive correlation between the rewiring rate and $w_{i}$, i.e., $r_{w_{i}}=\alpha_{1} w_{i}, \alpha_{1} \geq 0$. This is the case where a reliable node preferentially breaks its heavily loaded links with frequently interacted neighbors, especially in the case of cascading failures. The second design (Design 2) specifies the negative correlation between the rewiring rate and $w_{i}$, i.e., $r_{w_{i}}=\alpha_{2}\left(1-\frac{w_{i}}{\max \left\{w_{i}\right\}}\right), \alpha_{2} \geq 0$. This is the case where a reliable node preferentially breaks its infrequently used (or lightly loaded) links, especially for the purpose of alleviating interruptions to ongoing network operations.

The two example distributions of $\boldsymbol{W}$ are: $(a)$ exponential distribution (ED), and (b) log-normal distribution (LD). Both distributions are non-negative and suitable to describe the nonnegative link weights of adaptive weighted networks. For the purpose of fair comparisons between the strategies, the mean of the exponential distribution is set to be equal to that of the log-normal distribution. Given the same mean, denoted by $\langle w\rangle$, the two distributions have different dispersions, and so are the expectations of $w_{(1)}$ and $w_{(M)}$ under different distributions. Order statistics are exploited to evaluate $w_{(1)}$ and $w_{(M)}$, and in turn the lower bounds of $\tau^{*}$ in (23). This helps provide insight on the importance of dispersion on the reliability of the adaptive networks.

\section{A. Exponential Distribution}

The probability density distribution (PDF) and cumulative distribution function (CDF) of $w_{i} \forall i$ are $f\left(w_{i}\right)=\lambda e^{-\lambda w_{i}}$ and $F\left(w_{i}\right)=1-e^{-\lambda w_{i}}$, respectively. By exploring order statistics, the PDFs of $w_{(1)}$ and $w_{(M)}$ can be written as

$$
\begin{gathered}
f_{1}\left(w_{(1)}\right)=\lambda M e^{-\lambda M w_{(1)}}, \\
f_{M}\left(w_{(M)}\right)=\lambda M\left[1-e^{-\lambda w_{(M)}}\right]^{M-1} e^{-\lambda w_{(M)}} .
\end{gathered}
$$

We can find that $w_{(1)}$ has an exponential distribution with parameter $\lambda M$. As a result,

$$
\mathbb{E}\left[w_{(1)}\right]=\frac{1}{\lambda M}
$$

The PDF of $w_{(M)}$ can be rewritten as

$$
\begin{gathered}
f_{M}\left(w_{(M)}\right)=\lambda M\left[1-e^{-\lambda w_{(M)}}\right]^{M-1} e^{-\lambda w_{(M)}} \\
=\lambda M e^{-\lambda w-(M)} \sum_{i=0}^{M-1}\left(\begin{array}{c}
M-1 \\
i
\end{array}\right)\left(-e^{\left.-\lambda w_{(M)}\right)}\right)^{i} \\
=\sum_{i=0}^{M-1}(-1)^{i} M\left(\begin{array}{c}
M-1 \\
i
\end{array}\right) \lambda e^{-(i+1) \lambda w_{(M)},}
\end{gathered}
$$

By exploiting order statistics, the expectation of $\max _{i}\left\{w_{i}\right\}$, is given by

$$
\mathbb{E}\left[\max _{i}\left\{w_{i}\right\}\right]=\mathbb{E}\left[w_{(M)}\right]=\frac{1}{\lambda} \sum_{i=1}^{M} \frac{1}{i} .
$$

For Design 1 where $r_{w_{i}} \propto w_{i}, \mathbb{E}\left[\max _{i}\left\{r_{w_{i}}\right\}\right] \propto$ $\mathbb{E}\left[\max \left\{w_{i}\right\}\right]=\frac{1}{\lambda} \sum_{i=1}^{M} \frac{1}{i}$, the network is unreliable if

$$
\tau>\tau^{*} \propto \frac{\gamma+\frac{1}{\lambda} \sum_{i=1}^{M} \frac{1}{i}}{(k-1)\langle w\rangle} .
$$


For Design 2 where $r_{w_{i}} \propto \theta-w_{i}, \mathbb{E}\left[\max _{i}\left\{r_{w_{i}}\right\}\right] \propto \theta-$ $\mathbb{E}\left[\max \left\{w_{i}\right\}\right]=\theta-\frac{1}{\lambda} \sum_{i=1}^{M} \frac{1}{i}$, the network is unreliable if

$$
\tau>\tau^{*} \propto \frac{\gamma+\theta-\frac{1}{\lambda} \sum_{i=1}^{M} \frac{1}{i}}{(k-1)\langle w\rangle}
$$

\section{B. Log-normal Distribution}

Let $f\left(w_{i}\right)$ and $F\left(w_{i}\right)$ be the PDF and CDF of $w_{i}$. Then we have

$$
f\left(w_{i}\right)=\frac{1}{w_{i} \sigma \sqrt{2 \pi}} e^{-\frac{\left(\ln w_{i}-\mu\right)^{2}}{2 \sigma^{2}}}=\frac{\phi\left(\log w_{i}\right)}{w_{i}}
$$

and

$$
F(w)=\Phi\left(\log w_{i}\right),
$$

where $\mu$ and $\sigma$ are the mean and the standard deviation, respectively, and $\phi(\cdot)$ and $\Phi(\cdot)$ denote the PDF and CDF of the normal distribution respectively. The mean $m$ and the variance $v$ are functions of $\mu$ and $\sigma$, as given by

$$
\begin{aligned}
m & =e^{\mu+\frac{\sigma^{2}}{2}}, \\
v & =e^{\left(2 \mu+\sigma^{2}\right)}\left(e^{\sigma^{2}}-1\right) .
\end{aligned}
$$

By exploring order statistics, the PDF of $w_{(M)}$ can be written as

$$
\begin{aligned}
f_{M}\left(w_{(M)}\right) & =M\left[F\left(w_{(M)}\right)\right]^{M-1} f\left(w_{(M)}\right) \\
& =M\left[\Phi\left(\log w_{(M)}\right)\right]^{M-1} \frac{\phi\left(\log w_{(M)}\right)}{w_{(M)}} .
\end{aligned}
$$

The expectation of $w_{(k)}$ is

$$
\mathbb{E}\left[w_{(k)}\right]=\int_{0}^{+\infty} w_{(k)} f_{k}\left(w_{(k)}\right) d w_{(k)},
$$

for $k=1,2, \cdots, M$.

By submitting (29) to (30), we have $\mathbb{E}\left[w_{(M)}\right]$

$$
\mathbb{E}\left[w_{(M)}\right]=M \int_{-\infty}^{+\infty} e^{y}[\Phi(y)]^{M-1} \phi(y) d y,
$$

where $y=\log \left(w_{(M)}\right)$.

By exploiting order statistics, the expectation of $w_{(M)}$ is given by

$$
\mathbb{E}\left[w_{(M)}\right]=\frac{M}{\sqrt{\pi}}\left(\frac{1}{2}\right)^{M-1} \sum_{i=0}^{M-1}\left(\begin{array}{c}
M-1 \\
i
\end{array}\right) I(z \mid \mu, \sigma),
$$

where

$$
z=\frac{y-\mu}{\sqrt{2 \sigma}}
$$

$$
\begin{aligned}
& I(z \mid \mu, \sigma)= \\
& \left(\frac{2}{\sqrt{\pi}}\right)^{i}\left(\sum_{M_{1}, \cdots, M_{i}=0}^{+\infty} \frac{(-1)^{\sum_{l=1}^{i} M_{l}}}{\prod_{l=1}^{i}\left(2 M_{l}+1\right) \prod_{l=1}^{i} M_{l} !}\right)^{i} \times \\
& \quad \int_{-\infty}^{+\infty} e^{z^{2}+\sqrt{2} \sigma z+\mu} z^{2 \sum_{l=1}^{i} M_{l}+i} d z .
\end{aligned}
$$

For Design 1 where $r_{w_{i}} \propto w_{i}$,

$$
\mathbb{E}\left[\max _{i}\left\{r_{w_{i}}\right\}\right] \propto \frac{M}{\sqrt{\pi}}\left(\frac{1}{2}\right)^{M-1} \sum_{i=0}^{M-1}\left(\begin{array}{c}
M-1 \\
i
\end{array}\right) I(z \mid \mu, \sigma),
$$

the network is unreliable if

$$
\tau>\tau^{*} \propto \frac{\gamma+\frac{M}{\sqrt{\pi}}\left(\frac{1}{2}\right)^{M-1} \sum_{i=0}^{M-1}\left(\begin{array}{c}
M-1 \\
i
\end{array}\right) I(z \mid \mu, \sigma)}{(k-1)\langle w\rangle} .
$$

For Design 2 where $r_{w_{i}} \propto \theta-w_{i}, \mathbb{E}\left[\max _{i}\left\{r_{w_{i}}\right\}\right] \propto \theta-$ $\mathbb{E}\left[\max \left\{w_{i}\right\}\right]$

$$
=\theta-\frac{M}{\sqrt{\pi}}\left(\frac{1}{2}\right)^{M-1} \sum_{i=0}^{M-1}\left(\begin{array}{c}
M-1 \\
i
\end{array}\right) I(z \mid \mu, \sigma),
$$

the network is unreliable if

$$
\tau>\tau^{*} \propto \frac{\gamma+\theta-\frac{M}{\sqrt{\pi}}\left(\frac{1}{2}\right)^{M-1} \sum_{i=0}^{M-1}\left(\begin{array}{c}
M-1 \\
i
\end{array}\right) I(z \mid \mu, \sigma)}{(k-1)\langle w\rangle} .
$$

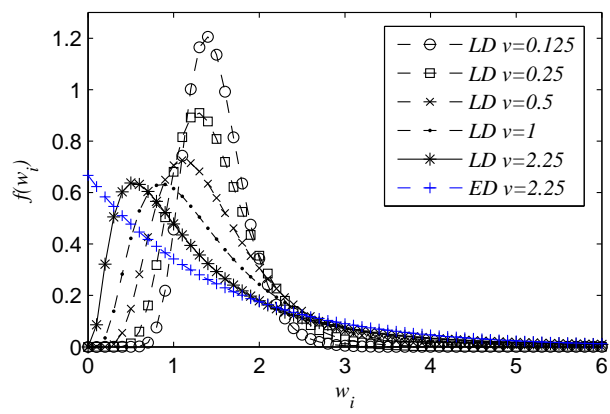

(a) The PDF of the log-normal distributions.

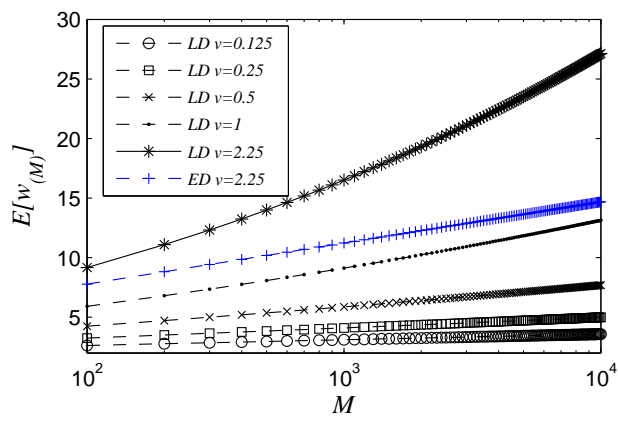

(b) $\mathbb{E}\left[w_{(M)}\right]$ of the log-normal distributions.

Fig. 2. The PDF and $\mathbb{E}\left[w_{(M)}\right]$ of the log-normal distribution, where the mean of the distribution is $m=1.5$, and $v$ is the variance of the distribution. We plot $v=0.125,0.25,0.5,1$ and 2.25 for the log-normal distribution to show the impact of the variance on the $\mathbb{E}\left[w_{(M)}\right]$.

We note that $\mathbb{E}\left[w_{(M)}\right]$ varies with different weight distributions. Fig. 2(a) plots the PDFs of the log-normal distributions under different variances $v$. Given the same mean, $m$, we can see that the log-normal distribution becomes increasingly dispersive, as $v$ increases. According to (27) and (30), Fig. 2(b) plots $\mathbb{E}\left[w_{(M)}\right]$ for the log-normal distribution with the growth of $M$. We see that, as the dispersion of the distribution increases, $\mathbb{E}\left[w_{(M)}\right]$ increases accordingly. Considering Design 
1 and 2, we can conclude that: (1) for Design 1, as $\mathbb{E}\left[w_{(M)}\right]$ gets larger, $\max \left\{r_{w_{i}}\right\}$ becomes larger and the threshold of $\tau$ that inhibits outbreaks becomes larger. Therefore, the more dispersive the distribution is, the more resistent the network is against outbreaks in Design 1; and (2) for Design 2, as $\mathbb{E}\left[w_{(M)}\right]$ gets larger, $\max \left\{r_{w_{i}}\right\}$ becomes smaller and the threshold of $\tau$ that inhibits outbreaks occur decreases. Therefore, the more dispersive the distribution is, the less resistent the network is against outbreaks in Design 2.

In addition to the reliability threshold $\tau^{*}$, another evaluation of the reliability of adaptive weighted networks against cascading failures is the steady-state density of unreliable nodes and the spreading speed of the infection/failures at an outbreak equilibrium of the adaptive weighted network. At a disease-free equilibrium point, $\left(\frac{d[I]_{w}}{d t}, \frac{d[S I]_{w}}{d t}\right)=(0,0)$, and $[I]=0$, and the cascading failure is inhibited. The population of infected nodes becomes zero. The entire population of nodes is healthy.

At an outbreak equilibrium, the average populations of susceptible (or healthy) and infected nodes stop changing over time, i.e., $\frac{d[I]}{d t}=0, \frac{d[S]}{d t}=0$, and $[I]>0$. As a result, the average number of links connecting different types of nodes, i.e., infected and susceptible nodes, stabilizes. $\frac{d[I I]_{w}}{d t}=0$, $\frac{d[S]_{w}}{d t}=0$, and $\frac{d[S S]_{w}}{d t}=0$. The populations of infected and healthy nodes are non-zero. By substituting these steady-state conditions into (8), we have

$$
\begin{aligned}
& \gamma[I]-\sum_{w} \beta_{w}[S I]_{w}=0 ; \\
& -\gamma[I]+\sum_{w} \beta_{w}[S I]_{w}=0 ; \\
& \left(\gamma+r_{w}\right)[S I]_{w}-\xi \frac{[S S]_{w}}{[S]} \sum_{w^{\prime}} \beta_{w^{\prime}}[S I]_{w^{\prime}}=0 ; \\
& \gamma[I I]_{w}-\beta_{w}[S I]_{w}-\xi \frac{[S I]_{w}}{[S]} \sum_{w^{\prime}} \beta_{w^{\prime}}[S I]_{w^{\prime}}=0 ; \\
& -\left(\gamma+\beta_{w}+r_{w}\right)[S I]_{w}+\gamma[I I]_{w} \\
& +\xi \frac{[S S]_{w}-[S I]_{w}}{[S]} \sum_{w^{\prime}} \beta_{w^{\prime}}[S I]_{w^{\prime}}=0 .
\end{aligned}
$$

By rewriting (33a) as $\sum_{w} \beta_{w}[S I]_{w}=\gamma[I]$ and then substituting into (33c) and (33d), we obtain

$$
\begin{aligned}
& \left(\gamma+r_{w}\right)[S I]_{w}-\xi \frac{[S S]_{w}}{[S]} \gamma[I]=0 \\
& \gamma[I I]_{w}-\beta_{w}[S I]_{w}-\xi \frac{[S I]_{w}}{[S]} \gamma[I]=0 .
\end{aligned}
$$

Since $[A B]=\sum_{w}[A B]_{w}, \forall A, B \in\{S, I\}$, we can rewrite (34) as

$$
\begin{aligned}
& \gamma[S I]+\sum_{w} r_{w}[S I]_{w}-\xi \frac{[S S]}{[S]} \gamma[I]=0 \\
& \gamma[I I]-\sum_{w} \beta_{w}[S I]_{w}-\xi \frac{[S I]}{[S]} \gamma[I]=0 .
\end{aligned}
$$

By substituting $\beta_{w}=\tau w, r_{w}=\alpha_{2}\left(1-\frac{w}{\max \{w\}}\right)$ (under Design 2), and $\sum_{w} \beta_{w}[S I]_{w}=\gamma[I]$ into (35), we can obtain

$$
\gamma[S I]+\alpha_{2}[S I]-\frac{\gamma \alpha_{2}}{\max \{w\} \tau}[I]-\xi \frac{[S S]}{[S]} \gamma[I]=0
$$

$$
\gamma[I I]-\gamma[I]-\xi \frac{[S I]}{[S]} \gamma[I]=0,
$$

which can be rearranged to provide the steady-state degrees of infected and healthy nodes, as given by

$$
\begin{aligned}
& {[S S]=\frac{\left(\alpha_{2}+\gamma\right)[S I]-\frac{\alpha_{2} \gamma}{\max \{w\} \tau}[I]}{\gamma([I I]-[I])}[S I]} \\
& {[I I]=[I]+\xi \frac{[S I]}{[S]}[I] .}
\end{aligned}
$$

By substituting (37) and $[I]+[S]=N$ into $2[S I]+[S S]+[I I]=$ $k N$, we can obtain

$2[S I]+\frac{\left(\alpha_{2}+\gamma\right)[S I]-\frac{\alpha_{2} \gamma}{\max \{w\} \tau}[I]}{\gamma([I I]-[I])}[S I]+[I]+\xi \frac{[S I][I]}{N-[I]}=k N$.

By substituting (37b), then (38) can be rewritten as

$2[S I]+\frac{\left(\alpha_{2}+\gamma\right)[S I]-\frac{\alpha_{2} \gamma}{\max \{w\} \tau}[I]}{\gamma \xi[I]}(N-[I])+[I]+\frac{\xi[S I][I]}{N-[I]}=k N$.

Together with constraints $[I]<N$ and $[S I]+[I I] \leq k N$, (39) provides the sufficient condition of the mass of infections/failures at an outbreak equilibrium of the networks. $\frac{[S I]+[I]]}{[I]}$ can be accordingly evaluated from (39) to show the degree of infected/failed nodes at the equilibrium, indicating changes in the topology of adaptive weighted networks in response to virus spread and cascading failures, as well as the effect of rewiring of weighted links.

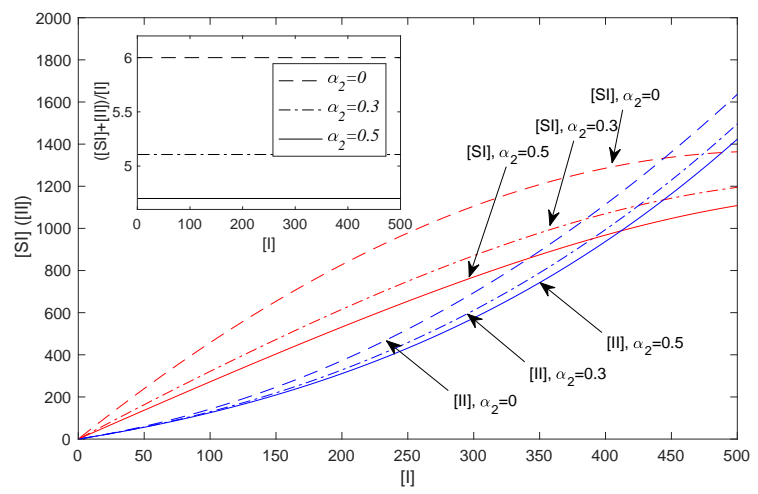

Fig. 3. The relations between $[I]$ and $[S I]$ (and $[I I]$ ). Plotted are the numbers of $[S I]$ (red) and $[I I]$ (blue) with respect to [I], under different values of $\alpha_{2}$. $N=1000, k=6, \tau=0.1, \gamma=0.5$, and $\max \{w\}=10$.

Fig. 3 plots $[S I]$ and $[I I]$ with the growth of $[I]$, where different values are tested for $\alpha_{2}$. We can see that $[S I]$ exhibits concavity with regards to $[I]$, while $[I I]$ exhibits convexity. In other words, the adaptive rewiring can increasingly isolate infected/failed nodes by breaking the links which can potentially infect healthy nodes. As a result, infections/failures become increasingly concentrated within the small set of infected/failed nodes. We also see that the average degree of infected/failed nodes remains consistent, as the growth of $[I]$ in an outbreak equilibrium, but the average degree does decrease with the growth of $\alpha_{2}$. Moreover, the average degree of infected/failed nodes is lower than the average degree of all nodes, indicating the infected/failed nodes are less connected and are prone to be separated from other nodes. 


\section{Numerical AND Simulation Result}

In this section, numerical and simulation results are provided to validate our proposed model and stability analysis. Figures are plotted based on discrete-time Monte-Carlo simulations of 100 iterations. Therefore, each data point in the figures is the average result of 100 independent runs. For each of the runs, a single infected node is randomly chosen at $t=0$, as the initial point of infection.

As discussed in Section VI, the rewiring process is intimately associated with the closeness between nodes. Here we analyze two different linear designs of $r_{w_{i}}$ : namely Design 1 with $r_{w_{i}}=\alpha_{1} w_{i}$; and Design 2 with $r_{w_{i}}=\alpha_{2}\left(1-\frac{w_{i}}{\max \left\{w_{i}\right\}}\right)$. In the simulations, only one of the designs is taken across the network. Two distributions of the link weights $w_{i}$ are compared: the exponential distribution and the log-normal distribution. For comparison fairness, the means of the exponential and the log-normal distributions are both set to be $1 / \lambda=m=1.5$ (so that the average value of $r_{w_{i}}$ is identical in both designs), and their variances are both set to be 2.25 (by configuring $m=e^{\mu+\sigma^{2} / 2}$ and $v=e^{2 \mu+\sigma^{2}}\left(e^{\sigma^{2}}-1\right)$ for the log-normal distribution; see (28) in Section VI). $\alpha_{1}$ and $\alpha_{2}$ are preconfigurable coefficients. We set $\alpha_{1}=0.2$ and $\alpha_{2}=0.3326$ to ensure the average value of $r_{w_{i}}$ is identical in both designs. In addition, we also plot the curves where the variance of the log-normal distribution is $v=0.125,0.25,0.5$ and 1 to show the impact of the variance on the propagation of cascading failure or virus spread. The simulations are carried out in an ER random network [43] of 1000 nodes connected by randomly generated 1997 links, where the weights of the links follow the exponential or log-normal distributions, ED and LD, as discussed in Section VI, respectively. For fair comparison, the distributions of $\boldsymbol{W}$ have identical mean $\langle w\rangle$. Other properties of the random network are summarized in TABLE I.

TABLE I

BASIC PROPERTIES OF THE RANDOM NETWORK WITH TWO EXEMPLARY DISTRIBUTIONS OF LINK WEIGHTS.

\begin{tabular}{c||rrrrr}
\hline Distribution & $v$ & $k$ & $\langle w\rangle$ & $w_{\min }$ & $w_{\max }$ \\
\hline & 0.125 & 3.994 & 1.5 & 0.6757 & 3.0985 \\
Log-normal $(L D)$ & 0.25 & 3.994 & 1.5 & 0.4082 & 4.4738 \\
& 0.5 & 3.994 & 1.5 & 0.2035 & 7.6161 \\
& 1 & 3.994 & 1.5 & 0.1579 & 10.2048 \\
& 2.25 & 3.994 & 1.5 & 0.0626 & 15.0701 \\
\hline Exponential $(E D)$ & 2.25 & 3.994 & 1.5 & 0.00085 & 15.296 \\
\hline
\end{tabular}

Fig. 4 plots the percentile of unreliable nodes $I$ with the growth of $\tau$ in the steady-state network, where both the two rewiring strategies are presented. We can see that $\tau^{*}$ increases in Design 1 as the dispersion of the link weights increases; see Fig. 4(a), and in Design 2, $\tau^{*}$ increases as the dispersion of the weights decreases; see Fig. 4(b). Moreover, the simulation results are consistent with the analysis in Section VI. Our analysis is validated with accuracy. As the weight distribution becomes more dispersive, the maximum value of the link weights in the network becomes increasingly larger, and the minimum value of the weights becomes smaller. To this end, the preferential disconnections of the links with frequently

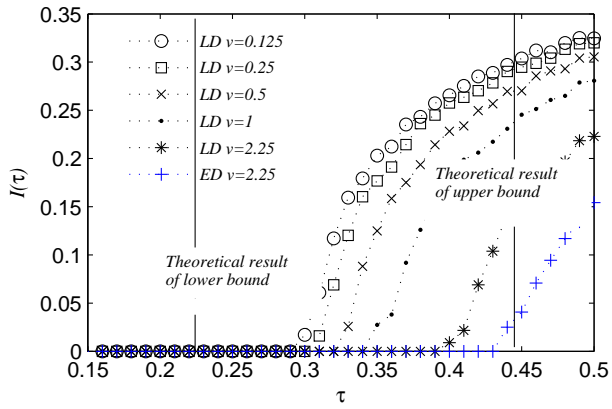

(a)

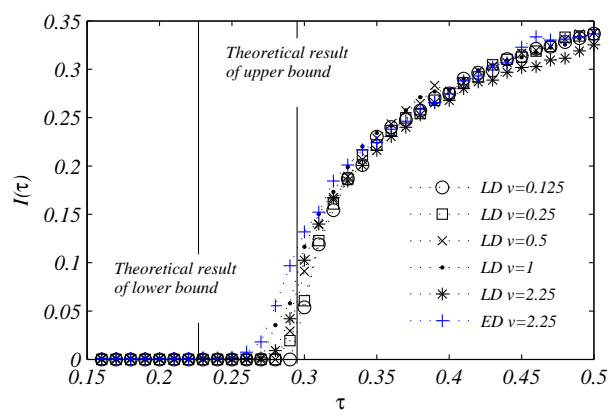

(b)

Fig. 4. The steady-state density of unreliable nodes $I$ as a function of $\tau$ under non-uniform rewiring rate, where (a) $r_{w_{i}}=\alpha_{1} w_{i}$, (b) $r_{w_{i}}=\alpha_{2}(1-$ $\frac{w_{i}}{\max \left\{w_{i}\right\}}$ ) with $\alpha_{1}=0.2$ and $\alpha_{2}=0.3326$.

communicated neighbors are likely to take place on the SI links with large weights, with the growth of the diversity of the weights. This can defer the outbreak of cascading failures or virus spread as the diversity of the weights grows in Design 1. On the contrary, in Design 2, the preferential disconnections of links with infrequently communicated neighbors are likely to take place on links with small weights, with the growth of the diversity of the weights. This can defer the outbreak of cascading failures or virus spread as the diversity of the weights decreases in Design 2. Furthermore, based on (22), the network can eventually become reliable if $\tau<0.223$ in Designs 1 and 2, consistent with the analytical results of the lower bound as shown in Fig. 4. Based on (23), the outbreaks occur if $\tau>0.445$ in Design 1 and $\tau>0.297$ in Design 2, consistent with our analytical results of the upper bound as shown in Fig. 4. In both designs, the network is reliable if $\tau$ is smaller than the analytical lower bound, and the network is unreliable if $\tau$ is larger than the analytical upper bound.

We note that our reliability analysis provides an upper bound for $\tau$ in reliable states (denoted by $\tau_{u}^{*}$ ) and a lower bound in unreliable states (denoted by $\tau_{l}^{*}$ ). Under the condition of $\tau<\tau_{u}^{*}$, the cascading failures can be eventually inhibited and the network is reliable; and under the condition of $\tau>\tau_{l}^{*}$, the cascading failures would proliferate and the network is deemed to be unreliable. These conditions are the sufficient conditions of the network reliability and unreliability, and may not be the necessary conditions. Confirmed by extensive simulations, we demonstrate that these sufficient conditions are effective, even though they can be loose in some circumstances, as shown for 
Design 1. In other circumstances, the sufficient conditions can be very tight, as shown for Design 2.

An interesting finding is that our designs can have a strong impact on the steady-state density of unreliable nodes in the network. In Fig 4(a), we see that the steady-state density of unreliable nodes decreases, as the dispersion of the link weights increases. In the case that the SI links with large weights are disconnected preferentially, only the SI links with small weights are left intact, leading to the reduction of the average transmission rate of the network. As a result, the steady-state density of unreliable nodes declines in Design 1. In contrast, in the case that the SI links with small weights are disconnected preferentially, the $S I$ links with large weights are left intact and this can increase the rate of turning reliable nodes to be unreliable. In other words, the steady-state density of unreliable nodes increases, as the dispersion of the weights grows. Finally, by assessing Fig. 4, we can notice that, given the same mean and variance, an exponential distribution of the links weights is preferred over the log-normal distribution in regards of network reliability under Design 1; and the other way around under Design 2.

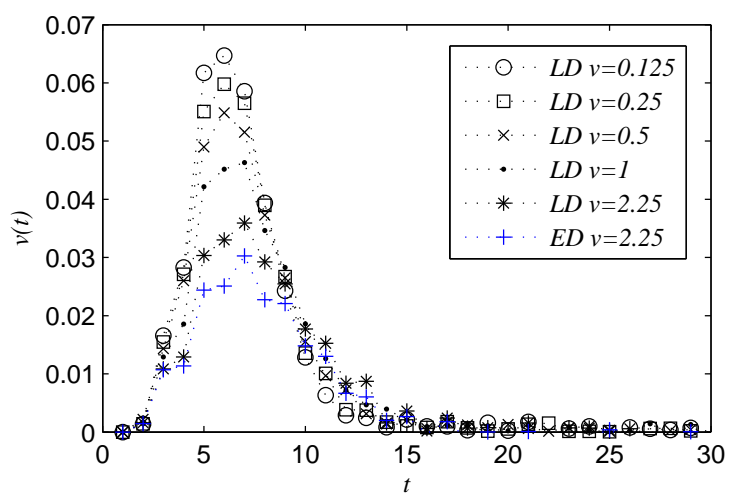

(a)

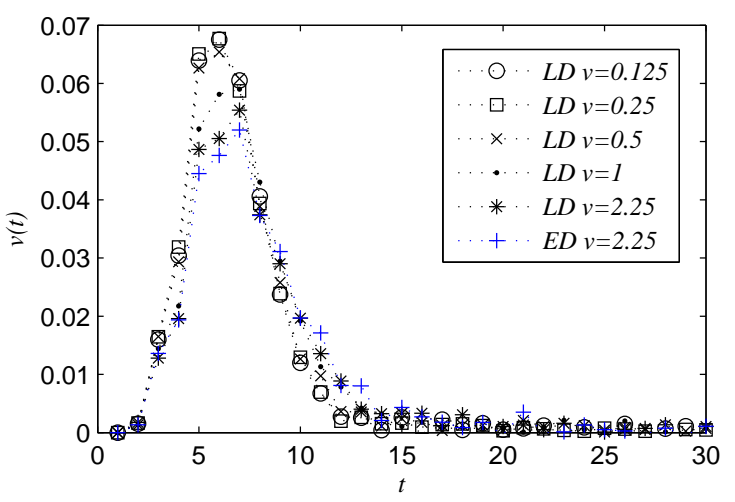

(b)

Fig. 5. The spreading velocity of infection $v(t)$ at each time slot $t$ under the two rewiring designs, where (a) Design 1: $r_{w_{i}}=\alpha_{1} w_{i}$, (b) Design 2: $r_{w_{i}}=\alpha_{2}\left(1-\frac{w_{i}}{\max \left\{w_{i}\right\}}\right)$, with $\alpha_{1}=0.2, \alpha_{2}=0.3326$ and $\tau=0.5$.

The spreading velocity of the virus or failure is an important measure of the designs. We define the spreading velocity as the difference of infection density between consecutive time slots, denoted by $v(t)=i(t)-i(t-1)$. Fig. 5 plots the spreading velocity under two designs, as the time elapses. We can see that in both designs, the more dispersive the distribution of the link weights is, the lower the velocity peak is. This indicates larger dispersion of the link weights can result in slower spreading. In Design 1, the disconnections of SI links with large weights remove the fast propagation paths of virus or failures, hence slowing down the propagation of virus or failures. And in Design 2, although the SI links with small weights are disconnected preferentially, the density of those links decreases as the dispersion of the link weights grows. As a result, large dispersion of the weights can also reduce the spreading velocity of virus or failures in Design 2.

In the special case where $r_{w_{i}}=r$, we can calculate the accurate value of $\tau$ by using (24). Fig. 6 shows the impact of the rewiring process and the weight distribution on the reliability threshold $\tau^{*}$ in the special case. The figure confirms the validity of the analytic results of $\tau^{*}$ from (24) by comparing with Monte-Carlo simulations. With the identical value of $\langle w\rangle$, we can see that the distribution of the weight $w_{i}$ has little impact on $\tau^{*}$, and hence validates our analysis. We also see that $\tau^{*}$ increases with the growth of the rewiring rate $r$. That is because, as the rewiring rate $r$ grows, the SI links can be increasingly likely to be disconnected. This leads to the reduction of the transmission paths. Therefore, the interruption of infection by the rewiring process can make the transmission increasingly difficult; or in other words, inhibits the transmission.

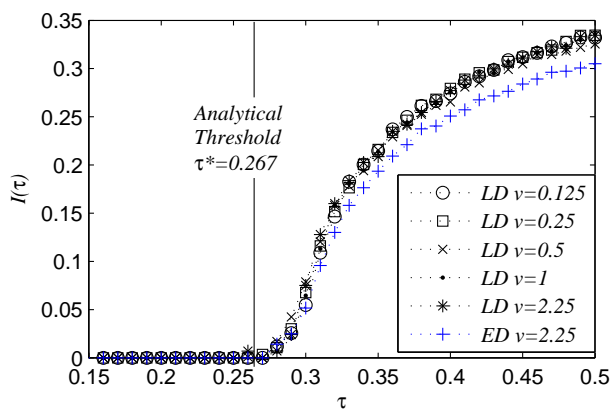

(a) The steady-state density of infected nodes $I$ as a function of $\tau$ in random networks, where $r=0.2, \gamma=1$.

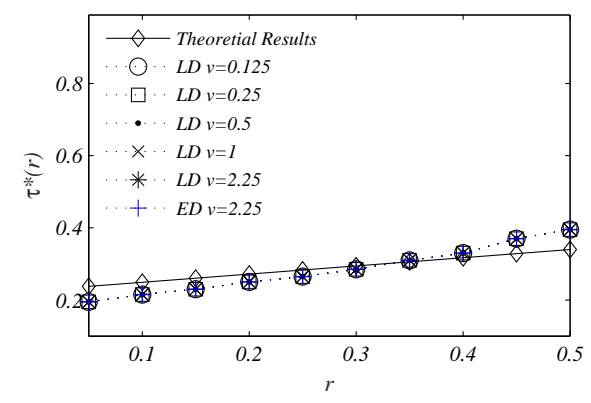

(b) The reliability threshold $\tau^{*}(r)$ as a function of rewiring rate $r$ in random networks.

Fig. 6. The special case of uniform rewiring rate, where the theoretical results of reliability threshold $\tau^{*}$ are given by (24).

In practice, networks can display a small-world effect [44] and a scale-free property [29]. These networks are particularly 
relevant to NFV. As a matter of fact, these networks have been widely used to portray actual virtual network characteristics [45-48]. It has also been proposed to construct virtual networks to comply with scale-free or small-world models, in attempts to reduce network average path length and to simplify NFV [46, 47]. In this sense, our simulation settings align with the virtual network characteristics. We proceed to carry out Monte-Carlo simulations on weighted networks with small-world effect and scale-free property, respectively. The properties of the two types of networks with 500 nodes connected by 1500 links are summarized in TABLE II. Fig. 7 shows the density of infected nodes $i(t)$ under the types of two sets of networks. It is clear that, in both WS small-world and $B A$ scale-free networks [29], [44], increasing the dispersion of the link weights can lead to a decline of the infected population in the steady-state network in Design 1, while decreasing the dispersion can do so in Design 2.

TABLE II

BASIC PROPERTIES OF WS NETWORK AND BA NETWORK

\begin{tabular}{c||c|rrrrr}
\hline Network & Distribution & $v$ & $k$ & $\langle\mathrm{w}\rangle$ & $w_{\max }$ & $w_{\min }$ \\
\hline \multirow{5}{*}{$\begin{array}{l}\text { WS } \\
\text { Network }\end{array}$} & Log-normal & 0.125 & 6 & 1.5 & 3.067 & 0.6365 \\
& & 0.25 & 6 & 1.5 & 3.4048 & 0.4512 \\
& & 0.5 & 6 & 1.5 & 6.5457 & 0.2993 \\
& & 1 & 6 & 1.5 & 8.4185 & 0.1915 \\
\cline { 2 - 7 } BA & Exponential & 2.25 & 6 & 1.5 & 10.5253 & 0.00081 \\
\hline \multirow{3}{*}{ Network } & & 0.125 & 6 & 1.5 & 3.1527 & 0.7015 \\
& & & 6 & 1.5 & 17.1523 & 0.0863 \\
\hline & & 0.25 & 6 & 1.5 & 4.4506 & 0.5291 \\
& & 0.5 & 6 & 1.5 & 6.4403 & 0.2548 \\
& & 1 & 6 & 1.5 & 8.4293 & 0.1284 \\
& Exponential & 2.25 & 6 & 1.5 & 15.2284 & 0.0694 \\
\hline
\end{tabular}

In general, our simulation results show that the reliability threshold $\tau^{*}$ depends on the distribution of the link weights and the specific rewiring strategy in the adaptive weighted networks. Preferentially disconnecting links to unreliable neighbors can effectively inhibit the spread of virus or failures, e.g., by increasing the reliability threshold, and reducing the steady-state population of unreliable nodes, and the spreading velocity of instability. The conclusion drawn is that the larger the dispersion of the link weights is, the more effectively the instability can be prevented from proliferation. On the other hand, preferential disconnections of the links with small weights can inhibit the spread as the dispersion of the weights decreases, e.g., increasing the reliability threshold and reducing the steady-state population of unreliable nodes. Unexpectedly, the dispersion of the link weights slows down the spread velocity as the links with small weights are preferentially disconnected.

\section{CONCLUSION}

In this paper, we proposed a mean-field approximated dynamic system to model the time-varying populations of failed nodes and risky links in adaptive weighted networks. A linear stability analysis was conducted upon the dynamic system, and the threshold was identified for the network to

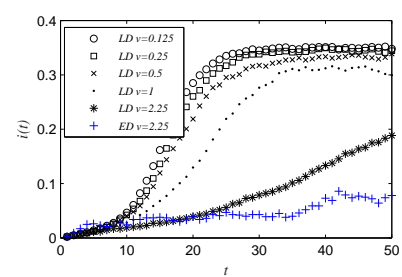

(a) Design 1

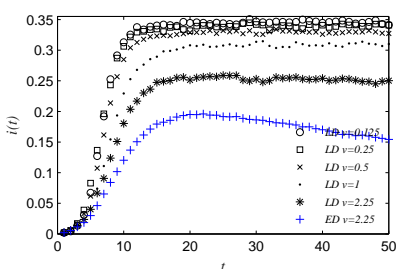

(c) Design 1

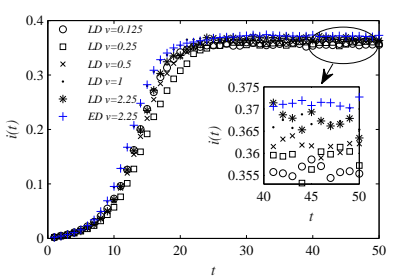

(b) Design 2

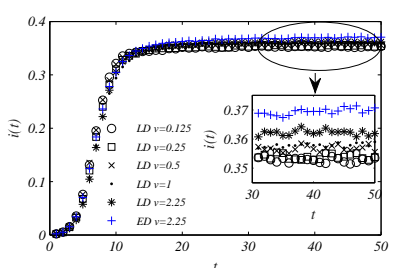

(d) Design 2
Fig. 7. The spread of virus or cascading failures in adaptive weighted networks with different rewiring designs, $\tau=0.3, \gamma=1$. The initial networks are $W S$ networks and $B A$ networks.

inhibit failures and remain reliable in the steady state. Validated by simulations, our analysis revealed that the threshold depends on both the distribution of the link weights and the adopted rewiring strategy. It is also shown that preferentially disconnecting frequently communicated, suspicious peers can effectively inhibit failures and virus spread. As cascading failures, DDoS [49], computer virus [49] and malware [50], can be potentially analyzed by using our analysis which is generic with an emphasis on theoretical insights and understanding. The presented analysis is not closely coupled with real behaviors of specific vulnerability exploration of particular attacks and viruses though. In the future, we will take the anatomy of different attacks into account and evaluate network reliability under specific types of attacks.

\section{REFERENCES}

[1] A. Alamer, Y. Deng, G. Wei, and X. Lin, "Collaborative security in vehicular cloud computing: A game theoretic view," IEEE Netw., vol. 32, no. 3, pp. 72-77, May 2018.

[2] B. Hayes, "Cloud computing," Commun. Acm, vol. 51, no. 7, pp. 9-11, July 2008.

[3] R. Mijumbi, J. Serrat, J. L. Gorricho, N. Bouten, F. D. Turck, and R. Boutaba, "Network function virtualization: State-ofthe-art and research challenges," IEEE Commun. Surveys Tut., vol. 18, no. 1, pp. 236-262, Firstquarter 2016.

[4] X. Zha, W. Ni, K. Zheng, R. P. Liu, and X. X. Niu, "Collaborative authentication in decentralized dense mobile networks with key predistribution," IEEE Trans. Inf. Forensics Security, vol. 12, pp. 2261-2275, Oct 2017.

[5] T. Gross and B. Blasius, "Adaptive coevolutionary networks: a review," J. Royal Society Interface, vol. 5, pp. 259-271, Mar. 2008.

[6] S. Trajanovski, D. Guo, and P. Mieghem, "From epidemics to information propagation: Striking differences in structurally similar adaptive network models," Phys. Rev. E, vol. 92, p. 030801, Sep 2015.

[7] V. Marceau, N. Pierre-André, L. Hébert-Dufresne, A. Antoine, and J. D. Louis, "Adaptive networks: Coevolution of disease and topology," Phys. Rev. E, vol. 82, p. 036116, Sep 2010.

[8] S. T. Zargar, J. Joshi, and D. Tipper, "A survey of defense mechanisms against distributed denial of service (ddos) flooding 
attacks," IEEE Commun. Surveys Tut., vol. 15, no. 4, pp. 20462069, March 2013.

[9] T. Gross, C. D’Lima, and B. Blasius, "Epidemic dynamics on an adaptive network," Phys. Rev. Lett., vol. 96, p. 208701, May 2006.

[10] L. B. Shaw and I. B. Schwartz, "Fluctuating epidemics on adaptive networks," Phys. Rev. E, vol. 77, p. 066101, Jun 2008.

[11] X. Chen, W. Ni, T. Chen, I. B. Collings, X. Wang, R. P. Liu, and G. B. Giannakis, "Distributed stochastic optimization of network function virtualization," in Proc. IEEE Glob. Commun. Conf., Dec 2017, pp. 1-6.

[12] J. Zhang, D. Zeng, L. Gu, H. Yao, and M. Xiong, "Joint optimization of virtual function migration and rule update in software defined nfv networks," in Proc. IEEE Glob. Commun. Conf., Dec 2017, pp. 1-5.

[13] J. Kong, I. Kim, X. Wang, Q. Zhang, H. C. Cankaya, W. Xie, T. Ikeuchi, and J. P. Jue, "Guaranteed-availability network function virtualization with network protection and vnf replication," in Proc. IEEE Glob. Commun. Conf., Dec 2017, pp. 1-6.

[14] H. Yang, M. Tang, and H. F. Zhang, "Efficient community-based control strategies in adaptive networks," New J. Phys., vol. 14, p. 123017, Dec 2012.

[15] R. Pastor-Satorras and A. Vespignani, "Epidemic dynamics and endemic states in complex networks," Phys. Rev. E, vol. 63, no. 2, p. 066117, May 2001.

[16] Q. C. Wu and F. Zhang, "Threshold conditions for SIS epidemic models on edge-weighted networks," Physica A: Statistical Mechanics and its Applications, vol. 453, pp. 77-83, Jul 2016.

[17] P. Rattana, K. B. Blyuss, K. T. Eames, and I. Z. Kiss, "A class of pairwise models for epidemic dynamics on weighted networks," Bulle. math. biol., vol. 75, pp. 466-490, Mar 2013.

[18] Y. Z. Zhou and Y. J. Xia, "Epidemic spreading on weighted adaptive networks," Physica A: Statistical Mechanics and its Applications, vol. 399, pp. 16-23, Apr 2014.

[19] C. Dong, Q. J. Yin, W. Y. Liu, Z. J. Yan, and T. Y. Shi, "Can rewiring strategy control the epidemic spreading?" Physica A. Statistical Mechanics and its Applications, vol. 438, pp. 169177, Nov 2015.

[20] Y. R. Song, G. P. Jiang, and Y. W. Gong, "Epidemic propagation on adaptive coevolutionary networks with preferential localworld reconnecting strategy," Chinese Phys. B, vol. 22, p. 040205, Apr 2013.

[21] B. B. Chopard and M. Droz, Cellular automata. Amsterdam, The Netherlands: Springer, 1998.

[22] I. Tunc and L. B. Shaw, "Effects of community structure on epidemic spread in an adaptive network," Phys. Rev. E, vol. 90, p. 022801, Aug 2014.

[23] G. Demirel, E. Barter, and T. Gross, "Dynamics of epidemic diseases on a growing adaptive network," Scientific Reports, vol. 7, p. 42352, 2017.

[24] L. Horstmeyer, C. Kuehn, and S. Thurner, "Network topology near criticality in adaptive epidemics," Phys. Rev. E, vol. 98, no. 4, p. 042313, 2018

[25] P. Zhu, Q. Zhi, Y. Guo, and Z. Wang, "Analysis of epidemic spreading process in adaptive networks," IEEE Trans. Circuits Syst. II, Exp. Brief, 2018.

[26] N. Sherborne, K. Blyuss, and I. Kiss, "Bursting endemic bubbles in an adaptive network," Phys. Rev. E, vol. 97, no. 4, p. 042306, 2018.

[27] W. Wang, M. Tang, H. F. Zhang, H. Gao, Y. Do, and Z. Liu, "Epidemic spreading on complex networks with general degree and weight distributions," Phys. Rev. E, vol. 90, p. 042803, Oct 2014.

[28] G. Yan, T. Zhou, J. Wang, Z. Q. Fu, and B. H. Wang, "Epidemic spread in weighted scale-free networks," Chinese Phys. Lett., vol. 22, p. 510, Feb 2005.

[29] A. L. Barabási and R. Albert, "Emergence of scaling in random networks," Science, vol. 286, pp. 509-512, Oct 1999.

[30] G. H. Zhu, G. R. Chen, X. J. Xu, and X. C. Fu, "Epidemic spreading on contact networks with adaptive weights," $J$. Theor Biol., vol. 317, pp. 133-139, Jan 2013.

[31] Y. Feng, L. Ding, Y. H. Huang, and L. Zhang, "Epidemic spreading on weighted networks with adaptive topology based on infective information," Physica A: Statistical Mechanics and its Applications, vol. 463, pp. 493-502, Dec 2016.

[32] M. Sun, H. Zhang, H. Kang, G. Zhu, and X. Fu, "Epidemic spreading on adaptively weighted scale-free networks," J. Math. Bios., vol. 74, no. 5, pp. 1263-1298, 2017.

[33] P. Hu, L. Ding, and T. Hadzibeganovic, "Individual-based optimal weight adaptation for heterogeneous epidemic spreading networks," Communications in Nonlinear Science and Numerical Simulation, vol. 63, pp. 339-355, 2018.

[34] X. Wang, W. Ni, K. F. Zheng, R. P. Liu, and X. X. Niu, "Virus propagation modeling and convergence analysis in large-scale networks," IEEE Trans. Inf. Forensics Security, vol. 11, pp. 2241-2254, Jun 2016.

[35] C. Jing, C. Wang, Z. Zhao, C. Kai, R. Du, and G. J. Ahn, "Uncovering the face of android ransomware: Characterization and real-time detection," IEEE Trans. Inf. Forensics Security, vol. PP, no. 99, pp. 1-1, 2017.

[36] D. Arrowsmith and C. M. Place, Dynamical systems: differential equations, maps, and chaotic behaviour. Chapman and Hall, London: CRC Press, 1992.

[37] I. Cohn, T. El-Hay, N. Friedman, and R. Kupferman, "Mean field variational approximation for continuous-time bayesian networks," J. Mach. Learn. Res., vol. 11, no. 5, pp. 2745-2783, Dec 2010.

[38] C. T. Bauch, "The spread of infectious diseases in spatially structured populations: An invasory pair approximation," Math. Bios., vol. 198, pp. 217-237, Dec 2005.

[39] A. M. Lyapunov, "The general problem of the stability of motion," Int. J. Control, vol. 55, pp. 531-534, Jun 1992.

[40] V. M. Preciado, M. Zargham, C. Enyioha, A. Jadbabaie, and G. J. Pappas, "Optimal resource allocation for network protection against spreading processes," IEEE Trans. Control Netw. Syst., vol. 1, no. 1, pp. 99-108, March 2014.

[41] P. Van den Driessche and J. Watmough, "Reproduction numbers and sub-threshold endemic equilibria for compartmental models of disease transmission," Math. bios., vol. 180, pp. 29-48, June 2002.

[42] C. D. Meyer, Matrix analysis and applied linear algebra. Siam, 2000, vol. 2.

[43] B. Bollobás, "Random graphs," in Modern Graph Theory. New York, USA: Springer, 1998, pp. 215-252.

[44] D. J. Watts and S. H. Strogatz, "Collective dynamics of smallworld networks," Nature, vol. 393, pp. 440-442, Jun 1998.

[45] M. Esch and E. Tobias, "Decentralized scale-free network construction and load balancing in massive multiuser virtual environments," in Proc. 6th Int. Conf. Coll. Comput. Netw., Appl. Works., Oct 2010, pp. 1-10.

[46] W. Jia, Y. Hu, G. Shou, X. Jin, and Z. Guo, "Constructing limited scale-free topologies for virtual networks," in Proc. 1st IEEE Int. Conf. Comput. Commun. Inte., Oct 2016, pp. 270274.

[47] R. Lin, B. Wu, Y. Zhao, H. Zou, and L. Liu, "Critical nodes detecting in virtual networking environment," in 2014 IEEE World Congress on Services, June 2014, pp. 317-322.

[48] R. Agarwal, A. Banerjee, V. Gauthier, M. Becker, C. Yeo, and B. S. Lee, "Achieving small-world properties using bio-inspired techniques in wireless networks," Comput. J., vol. 55, no. 8, pp. 909-931, August 2012.

[49] D. Brockmann and D. Helbing, "The hidden geometry of complex, network-driven contagion phenomena," Science, vol. 342, no. 6164, pp. 1337-1342, 2013.

[50] J. Balthrop, S. Forrest, M. E. Newman, and M. M. Williamson, "Technological networks and the spread of computer viruses," Science, vol. 304, no. 5670, pp. 527-529, 2004. 


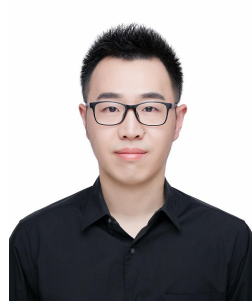

Bo Song received the B.E. degree from Liaocheng university, Liaocheng, China, in 2010 and the M.E. degree from Nanjing University of Posts and Telecommunications, Nanjing, China, in 2013. He is currently pursuing the Ph.D. degree with School of Cyberspace Security, Nanjing University of Post$\mathrm{s}$ and Telecommunications, Nanjing, China, and Global Big Data Technologies Centre, University of Technology, Sydney, Australia. His main research interests include graph theory, complex network, cyber security, and network dynamics.

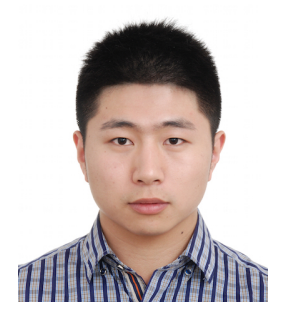

Xu Wang is currently pursuing the Ph.D. degree with School of Cyberspace Security, Beijing University of Posts and Telecommunications, Beijing, China, and Global Big Data Technologies Centre, University of Technology, Sydney, Australia. He received the B.S. degree from Beijing Information Science and Technology University, Beijing, China, in 2010. He visited CSIRO, Australia in 2014. His main research interests include graph theory, Markov theory, intrusion detection, cyber security, and blockchain.

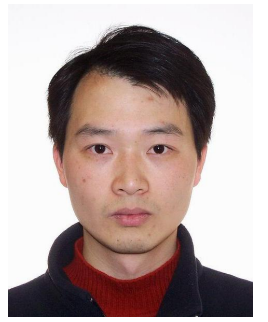

Wei Ni (M'09-SM'15) received the B.E. and Ph.D. degrees in Electronic Engineering from Fudan University, Shanghai, China, in 2000 and 2005, respectively. Currently, he is a Team Leader at CSIRO, Sydney, Australia, and an adjunct professor at the University of Technology Sydney (UTS) and Macquarie University (MQ). He also holds honorary positions at the University of New South Wales (UNSW). Prior to this, he was a postdoctoral research fellow at Shanghai Jiaotong University from 2005 - 2008; Deputy Project Manager at the Bell Labs R\&I Center, Alcatel/Alcatel-Lucent from 2005 - 2008; and Senior Researcher at Devices R\&D, Nokia from 2008 - 2009. His research interests include stochastic optimization, game theory, graph theory, as well as their applications to network and security.

$\mathrm{Dr} \mathrm{Ni}$ has been serving as the Vice Chair of IEEE NSW VTS Chapter and an Editor of IEEE Transactions on Wireless Communications since 2018, the secretary of IEEE NSW VTS Chapter from 2015 - 2018, Track Chair for VTCSpring 2017, Track Co-chair for IEEE VTC-Spring 2016, and Publication Chair for BodyNet 2015. He also served as Student Travel Grant Chair for WPMC 2014, a Program Committee Member of CHINACOM 2014, a TPC member of IEEE ICC'14, ICCC'15, EICE'14, and WCNC'10.

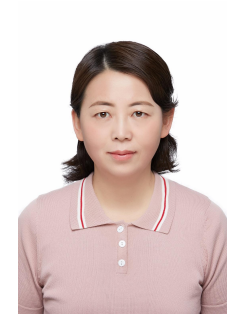

Yurong Song received B.S, Degree in Physcics from Qinghai Normal University, M.E degree in Computer application from East China Normal university in 2000, and Ph.D degree in Information \& Communication Engineering from Nanjing University of Posts \& Telecommunications in 2009 respectively. Currently she is a professor of college of Auotmation, Nanjing University of Posts \& Telecommunications. Her research interests include information propagation and its control strategies in complex networks; modeling, simulation and intelligent optimization of adaptive networks. She has published more than 50 international papers on complex networks.

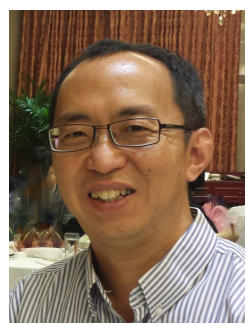

Ren Ping Liu (M'09-SM'14) received his B.E. and M.E. degrees from Beijing University of Posts and Telecommunications, China, and the Ph.D. degree from the University of Newcastle, Australia.

$\mathrm{He}$ is currently a Professor and Head of Discipline of Network \& Cybersecurity at University of Technology Sydney. Professor Liu is also the cofounder and CTO of Ultimo Digital Technologies Pty Ltd, developing IoT and Blockchain. Prior to that he was a Principal Scientist and Research Leader at CSIRO, where he led wireless networking research activities. He specialises in system design and modelling and has delivered networking solutions to a number of government agencies and industry customers. His research interests include wireless networking, Cybersecurity, and Blockchain.

Professor Liu was the founding chair of IEEE NSW VTS Chapter and a Senior Member of IEEE. He served as Technical Program Committee chairs and Organising Committee chairs in a number of IEEE Conferences. Prof Liu was the winner of Australian Engineering Innovation Award and CSIRO Chairman medal. He has over 150 research publications, and has supervised over $30 \mathrm{PhD}$ students.

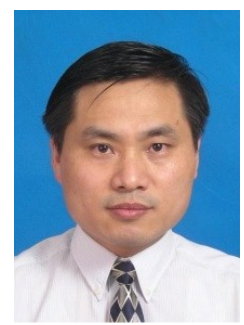

Guo-Ping Jiang (M'03) received the B.E. degree in electrical engineering from Hohai University, Nanjing, China, in 1988 and the Ph.D. degree in control theory and engineering from Southeast University, Nanjing, in 1997. From 1988 to 1992, he was an Assistant Teacher with the Department of Electrical Engineering, Hohai University. From March 1997 to July 2005, he was with the Department of Electronic Engineering, Nanjing University of Posts and Telecommunications, Nanjing, first as a Lecturer and then as an Associate Professor and a Professor. From June to September 2001, January to April 2002, and July to August 2005, he was with the Department of Electronic Engineering, City University of Hong Kong, Kowloon, Hong Kong, first as a Research Assistant and then as a Research Fellow. From August to December 2003, he was with the School of Quantitative Methods and Mathematical Sciences (QMMS), University of Western Sydney, Sydney, Australia, as a Visiting Fellow.

$\mathrm{He}$ has authored or coauthored more than 180 published articles in the area of nonlinear system and control. His current research interests include chaos synchronization and control, chaos-based communication, and complex dynamical networks. Prof. Jiang received the Awards for the New Century Excellent Talents of Ministry of Education, China, in 2006, and the Highlevel Talents of "Six Projects Sponsoring Talent Summits of Jiangsu Province, China, in 2008.

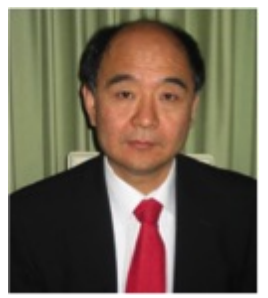

Y. Jay Guo (F'14) received a Bachelor Degree and a Master Degree from Xidian University in 1982 and 1984, respectively, and a Ph.D. Degree from Xian Jiaotong University in 1987, all in China. His research interest includes antennas, mm-wave and $\mathrm{THz}$ communications and sensing systems as well as big data technologies. He has published over 400 research papers and holds 24 patents in antennas and wireless systems. He is a Fellow of the Australian Academy of Engineering and Technology, a Fellow of IEEE and a Fellow of IET, and a member of the College of Experts of Australian Research Council (ARC). He has won a number of most prestigious Australian national awards, and was named one of the most influential engineers in Australia in 2014 and 2015.

Prof. Guo is a Distinguished Professor and the founding Director of Global Big Data Technologies Centre at the University of Technology Sydney (UTS), Australia. Prior to this appointment in 2014, he served as a Director in CSIRO for over nine years, directing a number of ICT research portfolios. Before joining CSIRO, he held various senior technology leadership positions in Fujitsu, Siemens and NEC in the U.K.

Prof. Guo has chaired numerous international conferences. $\mathrm{He}$ is the Chair Elect of International Steering Committee, International Symposium on Antennas and Propagation (ISAP). He was the International Advisory Committee Chair of IEEE VTC2017, General Chair of ISAP2015, iWAT2014 and WPMC2014, and TPC Chair of 2010 IEEE WCNC, and 2012 and 2007 IEEE ISCIT. He served as Guest Editor of special issues on "Antennas for Satellite Communications" and "Antennas and Propagation Aspects of 6090GHz Wireless Communications," both in IEEE Transactions on Antennas and Propagation, Special Issue on "Communications Challenges and Dynamics for Unmanned Autonomous Vehicles," IEEE Journal on Selected Areas in Communications (JSAC), and Special Issue on " $5 \mathrm{G}$ for Mission Critical Machine Communications," IEEE Network Magazine. 